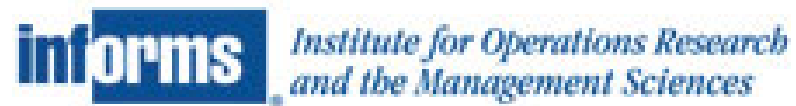

\author{
State Dependent Expected Utility for Savage's State Space \\ Author(s): Peter P. Wakker and Horst Zank \\ Source: Mathematics of Operations Research, Vol. 24, No. 1 (Feb., 1999), pp. 8-34 \\ Published by: INFORMS \\ Stable URL: http://www.jstor.org/stable/3690527 \\ Accessed: 19/04/2011 10:26
}

Your use of the JSTOR archive indicates your acceptance of JSTOR's Terms and Conditions of Use, available at http://www.jstor.org/page/info/about/policies/terms.jsp. JSTOR's Terms and Conditions of Use provides, in part, that unless you have obtained prior permission, you may not download an entire issue of a journal or multiple copies of articles, and you may use content in the JSTOR archive only for your personal, non-commercial use.

Please contact the publisher regarding any further use of this work. Publisher contact information may be obtained at http://www.jstor.org/action/showPublisher?publisherCode=informs.

Each copy of any part of a JSTOR transmission must contain the same copyright notice that appears on the screen or printed page of such transmission.

JSTOR is a not-for-profit service that helps scholars, researchers, and students discover, use, and build upon a wide range of content in a trusted digital archive. We use information technology and tools to increase productivity and facilitate new forms of scholarship. For more information about JSTOR, please contact support@jstor.org. 


\title{
STATE DEPENDENT EXPECTED UTILITY FOR SAVAGE'S STATE SPACE
}

\author{
PETER P. WAKKER AND HORST ZANK
}

\begin{abstract}
This paper generalizes the Debreu/Gorman characterization of additively decomposable functionals and separable preferences to infinite dimensions. The first novelty concerns the very definition of additively decomposable functionals for infinite dimensions. For decision under uncertainty, our result provides a state-dependent extension of Savage's expected utility. A characterization in terms of preference conditions identifies the empirical content of the model; it amounts to Savage's axiom system with P4 (likelihood ordering) dropped. Our approach does not require that a (probability) measure on the state space be given a priori, or can be derived from extraneous conditions outside the realm of decision theory. Bayesian updating of new information is still possible, even though no prior probabilities are given. The finding suggests that the sure-thing principle, rather than prior probability, is at the heart of Bayesian updating.
\end{abstract}

1. Introduction. Separability is one of the most important tools available for simplifying complex optimization, where several criteria have to be aggregated into one overall goal. Under some technical conditions, separability amounts to additive decomposability (Debreu 1960, Gorman 1968), and it has been used in many areas. Examples are decision under uncertainty (Savage 1954), consumer theory (Barten and Böhm 1982), interpersonal aggregation (Fleming 1952, Harsanyi 1955, Broome 1991), dynamic optimization (Strotz 1956, Koopmans 1972), and many other areas (Krantz et al. 1971, Keeney and Raiffa 1976). Debreu's theorem, as well as its many variations, only consider finitely many criteria. In many areas, the restriction to finitely many criteria is undesirable. It is often preferable to deal with infinitely many states of nature in decision under uncertainty, infinitely many persons in group aggregation, etc.

Whereas most preference representations in the literature are routinely extended from finite to infinite dimensions, no such extension has as yet been established for Debreu's result. Providing such an extension, for the special case of real-valued outcomes and monotonic preferences, is the purpose of this paper. The development of our new functional, additively decomposable on infinite-dimensional spaces, is similar to the definition of integrals and is also derived from approximations through step functions from above and below.

The research of this paper started with the search for a state-dependent generalization of Savage's (1954) expected utility, and a characterization thereof entirely in terms of endogeneous preference conditions. Probability is then no longer identifiable and the result is an additively decomposable functional. The state-dependent generalization of Savage (1954) is presented in Theorem 11. In view of this application, the main text of the paper is formulated for the context of decision under uncertainty. It could also have been formulated for interpersonal or intertemporal or other aggregations.

The outline of the paper is as follows. Section 2 surveys related literature on statedependent expected utility. It also points out that this paper only deals with cardinal state dependence. Ordinal state independence is preserved because utility for money is assumed increasing in every state. For economic applications, this restriction seems natural. Section 3

Received April 11, 1997; revised May 20, 1998.

AMS 1991 subject classification. Primary: 90A10.

OR/MS Index 1978 subject classification. Primary: Utility/Separability.

Key words. Separability, sure-thing principle, state-dependent utility, Bayesian updating, additive representation. 
discusses the absence of a general state-dependent expected utility functional in the literature thus far. In $\S 4$, elementary results are described for finite dimensions. State-dependent utility then coincides with additive conjoint measurement. Section 5 describes the difficulties for infinite state spaces. In particular, Example 4 motivates the specific form of our functional and shows why we do not adopt a completely general additive functional for infinite state spaces. Section 6 describes some natural preference conditions for state-dependent expected utility. The new functional is derived from those preference conditions. Section 7 demonstrates that the functional can be written as an integral if a countable additivity condition is added. Section 8 describes applications of our functional to updating, the characterizations of risk attitudes, and the elicitation of probability. Section 9 summarizes and concludes. Appendices A and B present mathematical modifications, and Appendices $C-G$, finally, present proofs.

2. State-dependent expected utility. The most famous result in decision under uncertainty was provided by Savage (1954), who presented preference conditions for subjective expected utility. Savage assumed that outcomes have a meaning and value independent of the state of nature with which they are associated. In several applications, however, it is undesirable to disentangle the value of an outcome from the associated state. An example is health insurance, in which the value of money is dependent on sickness or health. Other examples are described by Karni (1985) and Drèze (1987, Chapter 8). Therefore, several papers have dealt with state-dependent generalizations of Savage's model, where the utility of an outcome is permitted to depend on the state of nature with which it is associated.

A central issue in state-dependent expected utility is the nonidentifiability of probability. If utility can depend on the state of nature in any way, then probabilities are no longer uniquely determined. For identifying probability, most papers in the literature add exogeneous assumptions, invoking information other than observable choice. Examples are influence of the decision maker on probabilities and states and "idempotent" acts (Drèze 1961, 1987 Chapter 2), preferences between acts conditioned on different events (Luce and Krantz 1971, Fishburn 1973), hypothetical probabilities of states set by an experimentor (Karni, Schmeidler, and Vind 1983), lotteries with known probabilities over state-dependent outcomes belonging to different states (LaValle and Fishburn 1991, §5), preferences and utilities conditional on null states (Rubin 1949, 1987), and availability of some stateindependent outcomes (Karni 1993a, 1993b).

This paper focuses on a stage prior to the identifiability of probability. A general state-dependent extension of Savage's (1954) expected utility form is presented that is based solely on choice making and exhibits the characteristic inseparability of probability and utility. Our aim is therefore not to "resolve" the inseparability of probability and utility, but rather to accept it and incorporate it into a decision-model. Such an inseparability has been recommended by Kreps (1988, Formula 7.13), and appears in Fishburn (1970, Theorem 13.1), Rubin (1987), and Nau (1995). It has, however, not yet been presented for Savage's infinite-state model. It must be kept in mind that, without a given probability measure, no integration operation is available to define the functional form. To our knowledge, the only paper on state-dependent utility for infinite state spaces that does not invoke an integral is Rubin (1987). (A similar result for finite state spaces is Theorem 13.1 in Fishburn (1970).)

The paper in the literature closest to our work is Grodal and Mertens (1976), where probability and utility are also inseparable. They assume that an underlying countably additive measure on the state space is given a priori, with respect to which an absolute continuity condition is imposed. Thus, their functional can still be written as an integral form, contrary to our Theorem 11. In our Theorem 12, their result is extended by deriving the countably additive measure endogeneously, i.e., entirely from preference.

Let us come clean on one point. Our analysis also assumes an additional restriction. It 
concerns, however, the most common and relevant special case for economic science: We assume monetary outcomes and monotonicity and continuity. These conditions are entirely defined in terms of preferences. They imply state independence in an "ordinal" sense, i.e., outcomes are ordered the same way for different states of nature. Therefore, the analysis of this paper only addresses state dependence of utility in a cardinal sense. Example 4 describes a case of ordinal state dependence and indicates some of its unwarranted implications which further motivate our restriction to ordinal state independence.

There is another reason for the absence of a general state-dependent extension of Savage's expected utility in the literature. Many studies have restricted attention to linear utility. Whereas linearity sometimes refers to riskless outcomes themselves (Chateauneuf 1991, Nau 1995), it mostly refers to probabilistic mixing (Drèze 1961, 1987, Fishburn 1970, Theorem 13.1, Fishburn 1973, Karni, Schmeidler, and Vind 1983, Karni 1985, 1993a, Rubin 1987, LaValle and Fishburn 1991), in line with Anscombe and Aumann (1963). Under linear utility, the distinction between ordinal and cardinal state independence disappears: a linear function is an ordinal (increasing) transform of another linear function, if and only if it is a cardinal (linear) transform. Hence, if two linear state-dependent utility functions are ordinally equivalent, they are also cardinally equivalent. This explains how Anscombe and Aumann (1963) could obtain state-independence by only imposing an ordinal state-independence axiom (Assumption 1 there).

In Savage's model, there is no linear utility and P3 implies only ordinal, not cardinal, state independence. As pointed out by Karni (1993b, p. 433), it is primarily P4 (likelihood ordering; the only axiom violated in our Theorem 11) which implies cardinal state independence. A partial weakening of Savage's P4, leading to a partial degree of state dependence, is studied by Karni and Schmeidler (1993). Wakker (1987) adapted Karni, Schmeidler, and Vind's approach (1983) to continuous, as opposed to linear, utility. In his model, ordinal and cardinal state independence were distinguished.

In a mathematical sense, our analysis is related to Chew and Wakker (1996) and uses similar tools. There, an "outcome-dependent" capacity (nonadditive measure) was introduced that generalizes existing rank-dependent nonexpected utility theories by dropping the separation of utility and nonadditive probability. Similar rank-dependent forms appeared in Green and Jullien (1988), Quiggin (1989), and Segal (1993) for decision under risk.

An alternative extension of additive conjoint measurement to infinite product sets was studied by Vind (1990). His functional shares with ours the additivity property. It need not satisfy pointwise monotonicity and thus need not be constructable from limits of simple acts; this is illustrated in Example 4. His assumptions on domain are more restrictive than ours. For details see Appendix A, Observation 15(g) and the subsequent discussion.

The main characterizing preference condition in our model is the sure-thing principle. Other than that, only common preference axioms (weak ordering, monotonicity, continuity) are used. Our paper can, therefore, be considered a counterpart to Machina and Schmeidler (1992): They studied Savage's model in which, of the two critical expected-utility axioms, P4 (ensuring likelihood ordering) was kept and P2 (the sure-thing principle) was dropped. Under a strenghtening of P4, they then obtained a model for "probabilistic sophistication" that followed Savage in expressing uncertainties in terms of probabilities but, due to the dropping of $\mathrm{P} 2$, did not order probability distributions according to expected utility. Machina and Schmeidler's result was generalized by Epstein and LeBreton (1993), who used a weaker strengthening of Savage's P4 than Machina and Schmeidler in order to obtain an appealing interpretation as dynamic consistency. As compared to these works, we maintain Savage's P2 and drop P4. In other words, we abandon the existence of subjective probabilities and a likelihood ordering.

The preceding duality has a remarkable implication for updating, elaborated in $\S 8$. Machina and Schmeidler (1992) preserve the probability calculus of Bayesian updating in a nonexpected utility framework where updated preferences then depend on what would have 
TABLE 1. Existing and non-existing functionals

\begin{tabular}{lll}
\hline & Finite dimension & General (infinite) dim. \\
\hline Expected utility & $\sum_{j=1}^{n} p_{j} U\left(f\left(s_{j}\right)\right)(\mathrm{a} 1)$ & $\int_{s} U(f(s)) d P(\mathrm{~b} 1)$ \\
State-dep. expected utility & $\sum_{j=1}^{n} p_{j} U_{j}\left(f\left(s_{j}\right)\right)(\mathrm{a} 2)$ & $\int_{s} U_{s}(f(s)) d P(\mathrm{~b} 2)$ \\
Additive decomposability & $\sum_{j=1}^{n} V_{j}\left(f\left(s_{j}\right)\right)(\mathrm{a} 3)$ & $?(\mathrm{~b} 3)$ \\
\hline
\end{tabular}

happened outside the conditioning event. In our model, updating also results as a natural generalization of the traditional updating of expected utility, but the only point where it deviates is in the abandoning of prior probability. It preserves independence of updated preferences from counterfactual events, which is a foundation of the likelihood principle (Poirier 1988). According to the likelihood principle, the optimal estimate of an unknown parameter or the optimal decision to reject or accept a hypothesis is, given an observed value of a statistic, independent of what one would have done given other values of the statistic. The likelihood principle is central in Bayesian statistics and is preserved in our approach. In summary, our result suggests a central role for the sure-thing principle and a lesser role for prior probabilities in the normative debate on Bayesian updating. Prior probabilities simplify Bayesian updating but are not essential.

3. Absence of general state-dependent expected utility in the literature. To explain the absence, hitherto, of a general state-dependent extension of Savage's model, we first assume, contrary to Savage's model, that the state space is finite. In this case, general state-dependent expected utility is well-understood. Assume that the state space $S$ is $\left\{s_{1}, \ldots, s_{n}\right\}$. Let $f$ be an act, assigning outcome $f\left(s_{j}\right) \in \mathbb{R}$ to each state $s_{j}$. The expected utility formula of $f$ is given in Table 1 , Formula (a1), where $p_{j}$ is the probability of state $s_{j}$ and $U$ denotes utility. A first version of the state-dependent generalization is given in Formula (a2), where the subscript in $U_{j}$ indicates state-dependence. Any alternative state-dependent representation

$$
\sum_{j=1}^{n} q_{j} W_{j}\left(f\left(s_{j}\right)\right)
$$

with $p_{j} U_{j}=q_{j} W_{j}$ for all $j$ represents the same preferences and thus is empirically indistinguishable from the original representation. Hence only the products $p_{j} U_{j}$ are meaningful and it is preferable to rewrite the functional as in Formula (a3) where $V_{j}=p_{j} U_{j}$ for all $j$. That summation provides the state-dependent generalization of expected utility. As pointed out by Kreps (1988, Formula 7.13), state-dependent expected utility therefore is a special case of "additive conjoint measurement," axiomatized by Debreu (1960), Gorman (1968), Krantz, Luce, Suppes, and Tversky (1971), and others. The factoring out of probability $p_{j}$ and utility $U_{j}$ as in (a2) has little significance, i.e., probability is not identifiable under state-dependent expected utility. This point has often been discussed (Drèze 1961, 1987; Karni 1985, 1993a, 1993b, 1996; Rubin 1987; Kadane and Winkler 1988; Schervish, Seidenfeld, and Kadane 1990; Karni and Schmeidler 1993; Nau 1995). An empirical method for eliciting the product of probability and state-dependent utility has been described by Wakker and Deneffe (1996, end of §4).

State-dependent utility is more complicated for infinite state spaces. For these spaces, expected utility reads as in (b1) in the table, where $P$ denotes the probability distribution over $S$. State-dependent utility at first reads as (b2). Again, the same basic indeterminacy of probability and utility exists as in the finite state case, and alternative representations 


$$
\int_{S} W_{s}(f(s)) d Q
$$

can be chosen that represent the same preferences. For instance, let $Q$ have any positive density with respect to $P$, then divide $U_{s}$ by that density to obtain $W_{s}$. As for finite state spaces, one would like to drop the meaningless factorization into $P$ and $U_{s}$. However, a functional to drop the factorization from (b2) is not readily available in the literature, hence the question mark at (b3) in the table. Here, the analogy with finite state spaces stops.

Introducing the extension of an additively decomposable functional for infinitely many states, i.e., filling in Formula (b3), is our first task. The extension is not very difficult given ordinal state independence, and is defined by enclosure from above and below by simple acts, completely analogous to the definition of integrals. The absence in the literature of the required functional, and the desirability of developing it, was pointed out by Hübner and Suck (1993, p. 631 and concluding remarks). For related comments restricted to finite state spaces, see Fishburn (1970, Chapter 12).

4. Definitions and preliminary results. $S$ is a state space that can be finite or infinite. It is endowed with an algebra $\mathscr{A}$ of subsets, that is, $\mathscr{A}$ contains the universal event $S$, the complement $A^{c}$ relative to $S$ of each of its elements $A$, and, finally, the union $A \cup B$ of each pair of elements $A, B$. Subsets of $S$ contained in $\mathscr{A}$ are called events. For any partition of $S$ or of any subevent of $S$, it is always assumed without further mentioning that its elements are events. The outcome space is $\mathbb{R}$. (Connected topological outcome spaces are discussed in Appendix B.) An act is a bounded function from $S$ to the outcome space that is measurable, i.e., the inverse of every interval is an event. $\mathscr{F}$ denotes the set of all acts. Note that, without further mentioning, acts will be assumed to be bounded throughout this paper. A technique for dealing with unbounded acts is described by Wakker (1993).

For act $f$ and event $A, f_{A}$ denotes the restriction of $f$ to $A$. For acts $f, g$, and event $A, f_{A} g$ (abbreviating $f_{A} g_{A^{c}}$ ) denotes the act that agrees with $f$ on $A$ and with $g$ on $A^{c}$. Constant acts are sometimes identified with their associated outcomes. We may thus write, for outcome $x$, $f_{A} x$ for the act that agrees with act $f$ on event $A$ and is constant $x$ on $A^{c}$; the notation $x_{A} f$ is similar. For event $A, 1_{A}$ denotes the indicator function of $A$. For a finite partition $\left\{A_{1}, \ldots\right.$, $\left.A_{n}\right\}$ of $S, \sum_{j=1}^{n} x_{j} 1_{A j}$ denotes the act assigning $x_{j}$ to each $s \in A_{j}$, for each $j$. Such an act, taking only finitely many values, is a simple act. $\mathscr{F}^{s}$ denotes the set of all simple acts.

The preference relation is a binary relation $\geqslant$ on $\mathscr{F}$. It is a weak order if it is complete ( $f$ $\geqslant g$ or $g \geqslant f$ for all acts $f, g$ ) and transitive. The notation $>, \sim, \leqslant$, and $<$ is standard, i.e., $f>g$ if $f \geqslant g$ and not $g \geqslant f, f \sim g$ if $f \geqslant g$ and $g \geqslant f, f \leqslant g$ if $g \geqslant f$, and $f<g$ if $g$ $>f$. If there exists a representing function $V$ (i.e., $V$ is a real-valued function on the set of acts $\mathscr{F}$ such that $f \geqslant g \Leftrightarrow V(f) \geq V(g)$ for all acts $f, g)$, then $\geqslant$ is necessarily a weak order. Let us now summarize the structural assumptions made in the main body of this paper.

Assumption 1 (Structural Assumption). Acts are bounded measurable maps from the state space $S$ to the outcome space $\mathbb{R}$. The preference relation $\geqslant i$ is a binary relation on the set $\mathscr{F}$ of acts.

Simple acts play an important role in our analysis and several intermediate results will be formulated for simple acts. We will also see that several preference conditions can be restricted to simple acts. Together with some technical conditions, they then imply the corresponding conditions for the nonsimple acts. Event $A$ is null if $f_{A} g \sim g$ for all simple acts $f$ and $g$. Strict monotonicity holds if $x_{A} f>y_{A} f$ for all nonnull events $A$, simple acts $f$, and outcomes $x>y$.

The main condition of interest in our analysis is Savage's (1954) sure-thing principle. It requires 


$$
c_{I} f \geqslant c_{I} g \Leftrightarrow c_{I}^{\prime} f \geqslant c_{I}^{\prime} g
$$

for all acts $c, c^{\prime}, f, g$, and events $I$. The sure-thing principle holds on $\mathscr{F}^{s}$ if the condition is only imposed on all simple acts $c, c^{\prime}, f, g$. The condition is known under various names, such as (strong) separability in consumer choice theory (Barten and Böhm 1982), preferential independence in multiattribute utility theory (Keeney and Raiffa 1976), and (con)joint independence in conjoint measurement theory (Krantz et al. 1971).

If $S$ is finite, say $S=\left\{s_{1}, \ldots, s_{n}\right\}$, then $\mathscr{F}_{F}$ can be identified with $\mathbb{R}^{n}$ through the bijective mapping $f \rightarrow\left(f\left(s_{1}\right), \ldots, f\left(s_{n}\right)\right)$. Continuity of $\geqslant$ then means that, for each act $f,\{g: g$ $\geqslant f\}$ and $\{g: g \leqslant f\}$ are closed subsets of $\mathbb{R}^{n}$ with respect to the Euclidean topology. Debreu (1960) proved the following result, formulated here for the context of decision under uncertainty.

THEOREM 2 (DEBREU 1960, STATE-DEPENDENT EXPECTED UTILITY FOR FINITE STATE SPACES). Let the Structural Assumption 1 hold and let $S=\left\{s_{1}, \ldots, s_{n}\right\}$, where at least three states are nonnull. Then the following two statements are equivalent:

(i) There exist continuous functions $V_{j}: \mathbb{R} \rightarrow \mathbb{R}, j=1, \ldots, n$, that are strictly increasing for all nonnull states and constant for all null states, and such that $\geqslant$ is represented by

$$
V(f)=\sum_{j=1}^{n} V_{j}\left(f\left(s_{j}\right)\right) .
$$

(ii) $\geqslant$ is a continuous strictly monotonic weak order that satisfies the sure-thing principle. The following uniqueness holds for (i): $W(f)=\sum_{j=1}^{n} W_{j}\left(f\left(s_{j}\right)\right)$ represents $\geqslant$ if and only if there exist real numbers $\tau_{1}, \ldots, \tau_{n}$ and a positive $\sigma$ such that $W_{j}=\tau_{j}+\sigma V_{j}$ for all $j$, implying that $W=\tau+\sigma V$ for $\tau=\tau_{1}+\cdots+\tau_{n}$.

Strictly speaking, the uniqueness result in Theorem 2 may be considered slightly stronger than Debreu's (1960) because continuity of $W$ has not been presupposed but instead follows as a consequence (Wakker 1988). This generalized uniqueness result is used in what follows. The case of exactly one nonnull state is trivial; then there is no uncertainty. For two states of nature, an additional condition must be added in Statement (ii), for instance, the "hexagon condition" or the "Thomsen condition" (Karni and Safra 1998).

To obtain a.(state-independent) expected utility representation in Statement (i), and thus a finite-state version of Savage's (1954) result, the conditions in Statement (ii) must be strengthened. Such strengthenings have been provided by Grodal (1978), Wakker (1984, 1989), Nakamura (1990), and Gul (1992). An essential intuitive step in attaining such a separation of probability and utility is an identification, in at least a cardinal sense, of outcomes contingent on different states of nature. For further discussion and a clarifying example of this point, see Karni (1996).

5. Complications for infinite state spaces. Following Savage (1954), we now introduce a nonatomicity condition that implies infinity of $S$. An event $A$ is an atom if, for every subevent $B$ of $A$, either $B$ or $A \backslash B$ is null. We will assume that $S$ contains no atoms. That assumption is somewhat weaker than the "atomless" condition (see Appendix A) commonly adopted in the literature, but suffices for our purposes.

A major complication in the extension to infinite state spaces concerns the topological restrictions to be imposed. Continuity with respect to the product topology is too restrictive for our purposes. Therefore a "finite-dimensional" simple-continuity condition is imposed on $\mathscr{F}^{s}$ which requires that, for each finite partition $\left\{A_{1}, \ldots, A_{n}\right\}$ of $S$, the preference relation 
over the finite-dimensional subspace of acts of the form $\sum_{j=1}^{n} x_{j} 1_{A_{j}}$ is continuous. That is, for every $\left(x_{1}, \ldots, x_{n}\right) \in \mathbb{R}^{n},\left\{\left(y_{1}, \ldots, y_{n}\right) \in \mathbb{R}^{n}: \sum_{j=1}^{n} y_{j} 1_{A_{j}} \geqslant \sum_{j=1}^{n} x_{j} 1_{A_{j}}\right\}$ and $\left\{\left(y_{1}, \ldots\right.\right.$, $\left.\left.y_{n}\right) \in \mathbb{R}^{n}: \sum_{j=1}^{n} y_{j} 1_{A_{j}} \leqslant \sum_{j=1}^{n} x_{j} 1_{A_{j}}\right\}$ should be closed subsets of $\mathbb{R}^{n}$.

Proposition 3. Assume that $S$ contains no atoms and that $\geqslant$ is a simple-continuous strictly monotonic weak order that satisfies the sure-thing principle on $\mathscr{F}^{s}$. Then for each event $A$ there exists a continuous and either strictly increasing or constant function $V_{A}$ : $\mathbb{R} \rightarrow \mathbb{R}$ such that $\geqslant$ is represented on $\mathscr{F}^{s}$ by

$$
V\left(\sum_{j=1}^{n} x_{j} 1_{A_{j}}\right)=\sum_{j=1}^{n} V_{A_{j}}\left(x_{j}\right)
$$

The following uniqueness holds: $W\left(\sum_{j=1}^{n} x_{j} 1_{A_{j}}\right)=\sum_{j=1}^{n} W_{A_{j}}\left(x_{j}\right)$ represents $\geqslant$ on $\mathscr{F}^{s}$ if and only if there exist real numbers $\tau_{A}$ for each event $A$ and a positive $\sigma$ such that $W_{A}=\tau_{A}$ $+\sigma V_{A}$ for all $A$, where $\tau_{A \cup B}=\tau_{A}+\tau_{B}$ for all disjoint events $A, B$ and $W=\tau_{S}+\sigma V$.

The uniqueness result, up to multiplication by a positive "scale" factor $\sigma$ and addition of a signed bounded finitely additive measure $\tau$ is characteristic of state-dependent utility, and will return in later theorems.

Proposition 3 can be interpreted as a state-dependent extension of Savage's (1954) result for simple acts. Next we consider the extension to nonsimple acts. It turns out that, in the presence of weak ordering and the sure-thing principle, the technical conditions in the proposition, i.e., strict monotonicity and simple-continuity, do not suffice to ensure the desired representation. Loosely speaking, the sure-thing principle and strict monotonicity only impose restrictions within finite-dimensional subspaces of $\mathscr{F}_{\text {p }}$. Different finite-dimensional subspaces of $\mathscr{F}$ that share only the origin can be entirely unrelated. These claims are illustrated by the following example.

EXAmple 4. Assume that $S=[0,1]$, endowed with the regular Borel $\sigma$-algebra (the smallest $\sigma$-algebra containing all intervals) and the Lebesgue measure (assigning to each interval its length). The functional $V$ that represents $\geqslant$ is linear. On $\mathscr{F}^{s}, V$ is expected value. First a description is given of the extension of $V$ from $\mathscr{F}^{s}$ to some, but not all, nonsimple acts, for three different versions of the example. Then it is explained how $V$ can be extended to all nonsimple acts for each of the three versions. Finally, the example is discussed.

(Version A) For $f(s)=s+1, V(f)=-1$.

(VERSION B) Consider the partition $\pi=\{[0,1 / n),[1 / n, 2 / n), \ldots,[1-1 / n, 1),[1]\}$. Let $\mathscr{F}^{*}$ contain all acts that are 0 at all points $j / n$ and are linear on each interval of the partition. $\mathscr{F}^{*}$ is a linear space that intersects $\mathscr{F}^{s}$ only at the origin (the constant 0 act). Let $P^{*}$ be any arbitrary probability measure on $[0,1]$ and $U^{*}$ any continuous function from $\mathbb{R}$ to $\mathbb{R}$ such that $U^{*}(0)=0 ; U^{*}$ need not be increasing. Let $V$ be expected utility with respect to $P^{*}$ and $U^{*}$ on $\mathscr{F F}^{*}$.

(VERSION C) Let $\mathscr{F} *$ and $\mathscr{F}_{*} * *$ be two linear subspaces of $\mathscr{F}$ such that the linear space spanned by any two of $\mathscr{F}^{s}, \mathscr{F}^{*}$, and $\mathscr{F}^{* * *}$ intersects the third only at the origin. Let $P^{*}$ and $P^{* *}$ be arbitrary probability measures on $[0,1]$ and $U^{*}$ and $U^{* *}$ any continuous functions from $\mathbb{R}$ to $\mathbb{R}$ such that $U^{*}(0)=0=U^{* *}(0)$. Let $V$ be expected utility with respect to $P^{*}$ and $U^{*}$ on $\mathscr{F}^{*}$, and expected utility with respect to $P^{* *}$ and $U^{* *}$ on $\mathscr{F}^{* *}$.

In each of the three versions we can extend $V$ to a linear functional on all of $\mathscr{F}$ by the Hahn-Banach extension theorem (Dunford and Schwartz 1958). Linearity of $V$ implies the sure-thing principle because 


$$
\begin{aligned}
V\left(c_{I} f\right)-V\left(c_{I} g\right) & =V\left(c_{I} 0\right)+V\left(0_{I} f\right)-\left(V\left(c_{I} 0\right)+V\left(0_{I} g\right)\right)=V\left(0_{I} f\right)-V\left(0_{I} g\right) \\
& =V\left(c_{I}^{\prime} 0\right)+V\left(0_{I} f\right)-\left(V\left(c_{I}^{\prime} 0\right)+V\left(0_{I} g\right)\right)=V\left(c_{I}^{\prime} f\right)-V\left(c_{I}^{\prime} g\right) .
\end{aligned}
$$

Therefore, $V\left(c_{I} f\right)-V\left(c_{I} g\right)$ and $V\left(c_{I}^{\prime} f\right)-V\left(c_{I}^{\prime} g\right)$ have the same sign and the preference between $c_{I} f$ and $c_{I} g$ is the same as between $c_{I}^{\prime} f$ and $c_{I}^{\prime} g$. Because $V\left(x_{A} f\right)-V\left(y_{A} f\right)=(x$ $-y) V\left(1_{A}\right)>0$ for $x>y$ and $A$ nonnull, strict monotonicity also holds for the nonsimple acts.

A phenomenon occurs in (a) that is unwarranted from a decision theoretical viewpoint: $f(s)$ $>0$ for all states $s$, but still $V(f)<0$ and $f$ is strictly less preferred than 0 . Another unwarranted phenomenon occurs in both (b) and (c): the restrictions of $V$ (and, correspondingly, of $\geqslant$ ) to several subparts of its domain are completely unrelated. All three cases (a), (b), and (c) illustrate that $V$ on $\mathscr{F}^{s}$ does not restrict $V$ on other parts of its domain to a satisfactory degree.

A natural condition for preference functionals $V$ in decision under uncertainty, violated in (a) of the example, is pointwise monotonicity: if $f(s) \geq g(s)$ for all $s$, then $V(f) \geq V(g)$. Similarly, pointwise monotonicity for $\geqslant$ requires that $f \geqslant g$ if $f(s) \geq g(s)$ for all $s$ (compare Rubin 1987, Axiom 0).

We next turn to the extension of additivity. A functional $V: \mathscr{F}_{F} \rightarrow \mathbb{R}$ is additively decomposable over disjoint events, or additive for short, if for each event $A$ there exists a functional $V_{A}$ on the set of restrictions $f_{A}$ of acts $f$ to $A$ such that for each finite partition $\left\{A_{1}, \ldots, A_{n}\right\}$ of $S$,

$$
V(f)=\sum_{j=1}^{n} V_{A_{j}}\left(f_{A_{j}}\right) .
$$

It is important to note that $V_{A}\left(f_{A}\right)$ does not depend on the partitioning of $A^{c}$ and neither on the part of $f$ outside of $A$. Linear functionals are additive (define $V_{A}\left(f_{A}\right)=V\left(f_{A} 0\right)$ ). Also, the expected utility functional (Formula (b1) in Table 1) is additive (define $V_{A}$ $=\int_{A} U(f(s)) d P$ ). Additive functionals were previously studied by Vind (1990).

Additivity of $V$ immediately implies that the functionals $V_{A}$ are also additive in the sense that $V_{B \cup C}\left(f_{B \cup C}\right)=V_{B}\left(f_{B}\right)+V_{C}\left(f_{C}\right)$ for all disjoint events $B, C$. The following lemma shows that additivity on $\mathscr{F}^{s}$ is in agreement with the functional described in Proposition 3 (that imposes (3) only when the $f_{A_{j}}$ are constant).

LEMMA 5. $\quad V: \mathscr{F}^{s} \rightarrow \mathbb{R}$ is additive if and only if there exists, for each event $A$, a function $V_{A}^{*}: \mathbb{R} \rightarrow \mathbb{R}$ such that $V\left(\sum_{j=1}^{n} x_{j} 1_{A_{j}}\right)=\sum_{j=1}^{n} V_{A_{j}}^{*}\left(x_{j}\right)$.

Because of its importance, the following observation is displayed and its, elementary, proof is presented in the main text.

OBSERVATION 6. If $\geqslant$ can be represented by an additive functional then it satisfies the sure-thing principle.

Proof. Writing $R$ ("relevant") for the complement of event $I$ ("irrelevant"), we get

$$
V\left(c_{I} f\right)-V\left(c_{I} g\right)=V_{I}\left(c_{I}\right)+V_{R}\left(f_{R}\right)-\left(V_{I}\left(c_{I}\right)+V_{R}\left(g_{R}\right)\right)
$$

and

$$
V\left(c_{I}^{\prime} f\right)-V\left(c_{I}^{\prime} g\right)=V_{I}\left(c_{I}^{\prime}\right)+V_{R}\left(f_{R}\right)-\left(V_{I}\left(c_{I}^{\prime}\right)+V_{R}\left(g_{R}\right)\right)
$$


Dropping the terms $V_{I}\left(c_{I}\right)$ in the first equality and the terms $V_{I}\left(c_{I}^{\prime}\right)$ in the second, the same right-hand sides result. Hence the left-hand sides are also equal. Then they surely have the same sign, and that implies that the preference between $c_{I} f$ and $c_{I} g$ is the same as between $c_{I}^{\prime} f$ and $c_{I}^{\prime} g$.

Additivity does not preclude the phenomena described in Example 4 because the representing functional there was linear and thus additive. For infinite-dimensional extensions of additive conjoint measurement, there may be interest in general functionals, as in Example 4, that do not satisfy a pointwise monotonicity condition (Streufert 1995). For economic applications of decision under uncertainty with monetary outcomes, however, we think there is little empirical interest in such general functionals. Therefore, the following section follows a different route, avoiding the complications of Example 4.

6. State-dependent expected utility for infinite state spaces. In our extension of state-dependent expected utility to all acts in $\mathscr{F}$, the empirically most important conditions of additivity and the sure-thing principle will not be extended to all of $\mathscr{F}$ from the start, but will initially only be imposed explicitly on $\mathscr{F}^{s}$. Instead, continuity will be extended to all of $\mathscr{F}$. That ensures that the functional $V$ can be extended from $\mathscr{F}^{s}$ to $\mathscr{F}$ in the same way as integrals: Each nonsimple act is enclosed between dominating and dominated simple acts that converge to that act in supnorm. Because of continuity, the value of the nonsimple act is the limit of the values of the converging simple acts. Thus, $V$ is a "natural" extension of integrals. A crucial implication of supnorm continuity, underlying this procedure, is pointwise monotonicity (Lemma 9). We subsequently find, as a "bonus," that additivity and the sure-thing principle, imposed only on $\mathscr{F}^{s}$, do hold on all of $\mathscr{F}$ after all. Thus, a satisfactory version of state-dependent expected utility has been obtained.

Under the supnorm, the distance between two acts $f, g$ is $\sup _{s \in s}|f(s)-g(s)| . V$ is supnorm-continuous if $\{f \in \mathscr{F}: V(f) \geq \lambda\}$ and $\{f \in \mathscr{F}: V(f) \leq \lambda\}$ are supnorm-closed for all $\lambda \in \mathbb{R}$. Similarly, $\geqslant$ is supnorm-continuous if, for all acts $f$, the sets $\{g \in \mathscr{F}$ : $g \geqslant f\}$ and $\{g \in \mathscr{F}: g \leqslant f\}$ are supnorm-closed. It is well-known that supnorm-continuity is equivalent to the common continuity on $\mathbb{R}^{n}$, and thus supnorm-continuity implies simple-continuity. That supnorm-continuity is not overly restrictive may be further accentuated by its equivalence to continuity of utility under expected utility and also under the more general Choquet expected utility, introduced by Schmeidler (1989). This equivalence was demonstrated by Chew and Wakker (1996, Observation 2); it should be kept in mind here that our acts are assumed to be bounded.

A number of steps in the derivation of the main result, Theorem 11, are made explicit in the text. These steps make it clear that the state-dependent expected utility functional proposed here generalizes integrals in a natural manner. Henceforth, up to Theorem 11, it is assumed that:

AssumPTION 7. $S$ contains no atoms and $\geqslant i$ is a strictly monotonic supnorm-continuous weak order that satisfies the sure-thing principle on $\mathscr{F}^{s}$.

This assumption implies that Proposition 3 can be applied, yielding an additive representing functional $V$ on $\mathscr{F}^{s}$. The functional $V$ is first extended to all of $\mathscr{F}$, through "certainty equivalents." Next, the required conditions are derived for $V . \gamma \in \mathbb{R}$ is a certainty equivalent for act $f$ if $\gamma \sim f$.

\section{LEMMA 8. Under Assumption 7, there exists a certainty equivalent for each act $f$.}

Because of strict monotonicity (applied to the constant acts), there exists at most one certainty equivalent for each act. We extend the functional $V$ of Proposition 3 to the nonsimple acts by defining $V(f)=V(\gamma)$ where $\gamma$ is the certainty equivalent of $f$. This functional obviously represents preferences and, as shown in Lemma 19 in the Appendix, is 


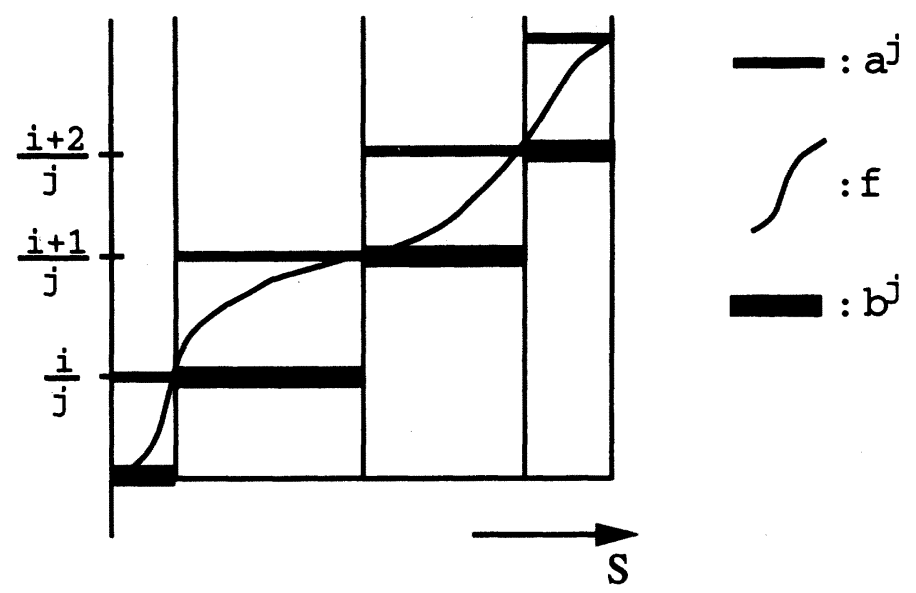

FIGURE 1. Enclosing $f$ from above and below by simple functions $a^{j}$ and $b^{j}$.

continuous. It obviously agrees with the definition on $\mathscr{F}^{s}$. An important observation is the following.

LEMMA 9. Under Assumption 7, the functional $V$ as just defined satisfies pointwise monotonicity.

Because of this lemma, the $V$ value of a nonsimple act $f$ results from $V$ values of simple acts in a similar fashion as an integral value of $f$ would. This can be seen as follows. It is well-known that there exist two sequences of simple acts $a^{j}$ and $b^{j}$ ( $a$ abbreviating "above" and $b$ "below") such that

$$
f(s)+1 / j \geq a^{j}(s) \geq a^{j+1}(s) \geq f(s) \geq b^{j+1}(s) \geq b^{j}(s) \geq f(s)-1 / j,
$$

for all $s$ and $j$ (see Figure 1). Then, because of pointwise monotonicity, $V(f)$ is enclosed increasingly tightly by $V\left(b^{j}\right) \leq V(f) \leq V\left(a^{j}\right)$. Because of supnorm-continuity (Lemma 19), $V\left(b^{j}\right)$ and $V\left(a^{j}\right)$ indeed converge to $V(f)$. The definition of the functional is similar to the construction of the Lebesgue integral. It does not invoke any structure on the state space other than measure-theoretical and in this sense is more general than the Riemann integral.

It will next be demonstrated that $V$ inherits additivity on $\mathscr{F}_{F}$ from additivity on $\mathscr{F}^{s}$. By Observation 6, this also ensures the sure-thing principle for all acts.

LEMMA 10. Under Assumption 7, $V$ as just constructed satisfies additivity.

At this point, the main theorem of this paper can be given.

Theorem 11 (State-dependent extension of SAVAge 1954). Let the Structural Assumption 1 hold and assume that $S$ contains no atoms. Then the following two statements are equivalent:

(i) $\geqslant$ is represented by a functional $V: \mathscr{F} \rightarrow \mathbb{R}$ that satisfies (1) additivity, i.e., there exist functionals $V_{A}$ defined on the restrictions of acts to $A$ such that

$$
V(f)=\sum_{j=1}^{n} V_{A_{j}}\left(f_{A_{j}}\right)
$$

for each finite partition $\left\{A_{1}, \ldots, A_{n}\right\}$ of $S$; (2) supnorm-continuity; (3) for each $A, V_{A}(x)$ (for outcome/constant act $x$ ) is either constant or strictly increasing in $x$. 
(ii) $\geqslant$ satisfies: (1) weak ordering; (2) strict monotonicity; (3) supnorm-continuity; (4) the sure-thing principle.

Furthermore, (i) implies pointwise monotonicity of $V$ and (ii) implies pointwise monotonicity of $\geqslant$. In (i), $V_{A}(x)$ is continuous in $x$ for each $A$.

The following uniqueness holds for (i): $W$ is additive $\left(W(f)=\sum_{j=1}^{n} W_{A_{j}}\left(f_{A_{j}}\right)\right.$ for any finite partition $\left\{A_{1}, \ldots, \dot{A}_{n}\right\}$ of $\left.S\right)$ and represents $\geqslant$ if and only if there exist real numbers $\tau_{A}$ for each event $A$ and a positive $\sigma$ such that $W_{A}=\tau_{A}+\sigma V_{A}$ for all $A$, where $\tau_{A \cup B}=\tau_{A}$ $+\tau_{B}$ for all disjoint events $A, B$ and $W=\tau_{S}+\sigma V$.

The theorem provides a state-dependent extension of Savage's expected utility. It does not provide a separation of probability and (state-dependent) utility, but instead it provides a decision-model where these factors are inextricably joined together.

7. Deriving an integral form under countable additivity. This section demonstrates that our functional can be written as an integral with respect to a countably additive measure $\mu$, under a stronger continuity condition. The result is given here for its mathematical convenience, not for its empirical content. The convenience results because the well-known integration techniques can now be invoked. The measure $\mu$ and the accompanying state dependent utility function $U_{s}$ are, however, only mathematical devises and do not represent additional empirical content. All relevant empirical information is contained in the general additively decomposable functional, without any measure $\mu$ or utility $U_{s}$ specified.

It is assumed in this section that the algebra $\mathscr{A}$ is a $\sigma$-algebra on $S$, i.e., it is closed under countable (instead of just finite) unions. Note that, in the special case of Theorem 11 where the functional $V$ can be decomposed into a probability measure $P$ and a utility function $U$, our axioms only imply finite additivity of $P$. The following continuity condition reinforces supnorm continuity to the effect of also implying countable additivity.

$\geqslant$ satisfies pointwise continuity if a sequence of "uniformly bounded acts that converge pointwise to an act also converge preference-wise to that act." A sequence of acts $f^{j}$ is uniformly bounded if there exist outcomes $x, y$ such that $x \geq f^{j}(s) \geq y$ for all $j$ and $s$, and it converges pointwise to $f$ if $\lim _{j \rightarrow \infty} f^{j}(s)=f(s)$ for all $s$. It converges preference-wise to $f$ if, for each act $g, g>f$ implies existence of a $J \in I N$ such that $g>f^{j}$ for all $j>J$ and $g<f$ implies existence of a $J \in I N$ such that $g<f^{j}$ for all $j>J$. Pointwise continuity of the functional $V$ is defined likewise: If a sequence of uniformly bounded acts converge pointwise, then their $V$ value also converges to the $V$ value of their limit. Pointwise continuity implies supnorm continuity (Lemma 20) and countable additivity (Lemma 22). It is weaker than (i.e., implied by) continuity of the state-dependent utility functions $U_{s}$ in (i) in the theorem (Lemma 27), hence is not overly restrictive.

THEOREM 12 (STATE-DEPENDENT EXPECTED UTILITY UNDER COUNTABLE ADDITIVITY). Let the Structural Assumption 1 hold and assume that $S$ contains no atoms. Then the following two statements are equivalent:

(i) There exists a countably additive measure $\mu$ on $S$ and for each state $s$ a strictly increasing (state-dependent utility) function $U_{s}: \mathbb{R} \rightarrow \mathbb{R}$ such that $\geqslant$ is represented by the pointwise continuous integral

$$
f \mapsto \int_{S} U_{s}(f(s)) d \mu
$$

(ii) $\geqslant$ satisfies: (1) weak ordering; (2) strict monotonicity; (3) pointwise continuity; (4) the sure-thing principle.

The following uniqueness holds for (i): $\mu,\left(U_{s}\right)_{s \in S}$ can be replaced by $\mu^{*},\left(U_{s}^{*}\right)_{s \in S}$ if and 
only if $\mu$ and $\mu^{*}$ have the same null events and $U_{s}^{*}=\tau(s)+\sigma \delta(s) U_{s}$ for all $s$ except on a $\mu$ null event, where $\tau$ is a measurable function from $S$ to $\mathbb{R}, \sigma$ is a positive constant, and $\delta$ is the Radon-Nikodym density function of $\mu$ with respect to $\mu^{*}$.

The integral in (i) is well-defined and real-valued for each act $f$. It is crucial for the uniqueness result regarding $\mu$ that each state-dependent utility is required to be strictly increasing. That excludes the case in which $\mu(A)>0$ but $A$ is null because of constant utility at $A$. Hence, $\mu$ identifies null events (Lemma 26). Statement (i) obviously implies pointwise monotonicity of $V$, and Statement (ii) of $\geqslant$, as in Theorem 11 . Theorem 12 deviates from Grodal and Mertens' (1976) result mainly because the measure $\mu$ is not given a priori but is determined endogeneously, completely in terms of preferences.

8. Applications. This section presents some applications of Theorem 11. We first demonstrate, on the basis of suggestions by Karni, Schmeidler, and Vind (1983) and Karni (1996), that updating through adjusted likelihoods ratios, the corner stone of Bayesian statistics, is still possible. Thus, Bayesian updating is extended from expected utility to state-dependent expected utility.

As a preparation, the classical updating from Bayesian statistics, which will be generalized hereafter, is described within the expected utility model. Let $f \mapsto \int_{s} U(f(s)) d P$ represent the prior preferences over acts, for a subjective probability measure $P$ and a (subjective) utility function $U$. Assume that we observe a value $X$ of some "statistic." The probability (density) for this observation is different for different states of nature, i.e., it is a function of the state of nature. This function is the likelihood function.

To avoid some technical complications, the likelihood function is assumed to take only finitely many values and is further assumed to be constant on each element $A_{j}$ of a partition $\left\{A_{1}, \ldots, A_{n}\right\}$. In other words, the observation of $X$ helps to distinguish between the "hypotheses" $A_{1}, \ldots, A_{n}$; conditional on any $A_{j}$, however, the observation $X$ does not provide further information. Say the likelihood of the observation is $\lambda_{j}$ for each $A_{j}$. Then, after the observation the probability for any $A_{j}$ is changed by a factor proportional to $\lambda_{j}$ according to the formula of Bayes. The updated preference relation, denoted $\tilde{\geqslant}$, can be represented by $f \mapsto \sum_{j=1}^{n} \lambda_{j} \int_{A j} U(f(s)) d P$; note here that normalization is not necessary for the representation of preferences.

The previously-discussed method for updating preference is first extended to statedependent utility when prior probabilities are known. Let the prior preferences be represented by $f \mapsto \int_{s} U_{s}(f(s)) d P$. With prior probability $P$ given, we can still apply Bayes' formula in the classical manner, and posterior preferences are represented by

$$
f \mapsto \sum_{j=1}^{n} \lambda_{j} \int_{A_{j}} U_{s}(f(s)) d P
$$

This manner of updating can be inferred from Karni, Schmeidler, and Vind (1983, Lemma); see also Karni (1996, §3.3), Karni and Schmeidler (1993), and Nau (1995, §7). Karni, Schmeidler, and Vind also demonstrate that an alternative equivalent state-dependent representation $f \mapsto \int_{S} W_{s}(f(s)) d Q$ for prior preference can be similarly updated into

$$
f \mapsto \sum_{j=1}^{n} \lambda_{j} \int_{A_{j}} W_{s}(f(s)) d Q
$$

This updated form represents the same posterior preferences as the updated form in (4). In 
other words, alternative representations for state-dependent expected utility remain indistinguishable under updating. From that observation, only one further step is required to extend the updating to our functional in which there is no factorization into probability and utility. If prior preferences are represented by an additive functional $V(f)$, then updated preferences are represented by

$$
f \mapsto \sum_{j=1}^{n} \lambda_{j} V_{A_{j}}\left(f_{A_{j}}\right)
$$

Normalization can be obtained by letting the functional assign value 0 to the act that is constant 0 and value 1 to the act that is constant 1 . The sure-thing principle is at the heart of this updating method, and prior probabilities play no role.

Our updating method is characterized for state-dependent expected utility with a subjective likelihood function. Likelihood is not objective and given a priori but is determined in terms of the decision maker's preferences. A topic for future research is under what conditions such subjective likelihoods coincide with objective likelihoods if the latter are available.

The preference relation $\geqslant$ again designates "prior" preferences, relevant before the receipt of information. Posterior preferences are indicated by a wiggle. Thus, $\tilde{\sim}, \tilde{>}, \tilde{\xi}$, and $\tilde{<}$ relate to posterior preferences and are derived from $\tilde{\geqslant}$ as the related symbols are from $\geqslant$. An observation is informative with respect to a partition $\left\{A_{1}, \ldots, A_{n}\right\}$ if, for each $j$, either $A_{j}$ is a null event for $\tilde{\geqslant}$ ("impossible a posterior"), or $f_{A_{j}} c \geqslant g_{A_{j}} c$ if and only if $f_{A_{j}} c \geqslant g_{A_{j}} c$, for all acts $f, g, c$. This means that the information does not distinguish between subevents of any $A_{j}$. In other words, given any $A_{j}$ nothing changes. The information only concerns the mutual likelihoods ratios of the different events ("hypotheses") $A_{j}$ in the partition. A case in which the condition is satisfied is when, given $A_{j}$, the true state of nature in $A_{j}$ is determined by a random mechanism (such as repeated coin tosses) unrelated to the observation.

THEOREM 13. Assume that the prior preferences are denoted by $\geqslant$ and that preferences are updated into $\geqslant$ after some observation. Assume that both preference relations satisfy the conditions and statements of Theorem 11 (for the same state and act space). Then the following two statements are equivalent for a partition $\left\{A_{1}, \ldots, A_{n}\right\}$ of the state space:

(i) There exist nonnegative numbers $\lambda_{j}$ such that the representation of Theorem 11 holds for $\geqslant$, and $f \mapsto \sum_{j=1}^{n} \lambda_{j} V_{A_{j}}\left(f_{A_{j}}\right)$ represents the updated preferences $\geqslant$.

(ii) The observation is informative with respect to the partition $\left\{A_{1}, \ldots, A_{n}\right\}$.

The theorem has demonstrated that Bayesian updating is possible even if a convenient decomposition $V_{A_{j}}=P\left(A_{j}\right) U$ is not available. Prior probability, the target of much criticism, apparently is not crucial for Bayesian updating.

We next turn to a study of risk attitudes. For finite state spaces, similar result were presented by Miyamoto and Wakker (1996). Constant risk aversion holds if $f \sim x \Leftrightarrow f$ $+\epsilon \sim x+\epsilon$ whenever $\epsilon>0$, for all acts $f$, outcomes $x$, and positive $\epsilon$.

THEOREM 14. Assume that the conditions and statements of Theorem 11 hold. Then constant risk aversion holds if and only if expected utility holds and $U(x)=a+b e^{c x}$ for some constants $a, b, c$ with $b c>0$, or $U(x)=a+b x$ for some constants $a, b$ with $b$ $>0$.

Similar results can be proved for positive outcomes and proportional ("relative") risk aversion. They follow from Theorem 14 through the replacement of all outcomes by their logarithms. We conjecture that Theorem 14, with state-dependent utility assumed but state-independent utility implied (and, of course, more general utility functions), holds true if we weaken constant risk aversion to decreasing risk aversion. That conjecture can be proved if Wakker (1989, Conjecture VII.6.10) is true. 
Theorem 14 and the results subsequently suggested are negative for state-dependent expected utility because common assumptions regarding risk attitude are simply not possible under state dependence. The model necessarily reduces to expected utility. Apparently, the common conditions for risk attitude need to be redefined for state-dependent utility. Alternative definitions were proposed by Karni (1983).

We finally discuss an idea of Karni (1993a, 1993b), for identifying probability. Assume that acts $a, b$ are given and suppose that, on the basis of prior assumptions, the state-dependent utility of $b$ must be 0 and the state-dependent utility of $a$ must be 1 , for all states. Then probabilities are directly identified in Theorem 11 through $P(A)=\left(V\left(a_{A} b\right)\right.$ $-V(b)) /(V(a)-V(b))$ for each event $A$. Note that the information on utilities of $a$ and $b$ is not derived from preference but is exogeneous. The identification of subjective probability just described is related to the method of Drèze $(1987, \S 2.8 .3)$, where moral hazard and "idempotent" acts are used to identify constant-valuation acts, Karni (1993a, 1993b, 1997), where the range (thus the highest and lowest value) of utility across different states is assumed identical, Nau and McCardle (1991) and Nau (1995), where avoidance of arbitrage implies the existence of "risk-neutral probabilities" which are defined relative to the market currency, the method of Karni and Schmeidler (1993), where the assumption is reduced to a differential form, i.e., the utility of 0 is 0 for all states, and has the same derivative at 0 for all states, the method of Maher (1993, Chapter 8) where two outcomes with state-independent utility are assumed, and, finally, Klibanoff (1995), who justifies the assumption of equally-valued outcomes in a dynamic setup with opportunity sets as outcomes.

The derivation of a state-dependent utility function from the preceding assumptions is a topic for future research. It requires measure-theoretical axioms regarding the role of null events and adaptation of the Radon-Nikodym theorem to finitely additive measures, subjects that are outside the scope of this paper.

If only two outcomes are given instead of a continuum as in our analysis, then the additively decomposable functional reduces to a finitely additive measure. For that case, many advanced results have been obtained (Fishburn 1986, 1992).

This paper has used the framework of decision under uncertainty, in which the functional represents state-dependent expected utility. The functional can be used in other areas as well. In dynamic optimization (Strotz 1956, Koopmans 1972), it generalizes discounted utility by allowing utility to depend on time. In welfare theory, it permits utilitarianism where utility depends on the individual (Harsanyi 1955), but no lotteries over income need be invoked. It thus extends Fleming (1952), an appealing but not very well-known predecessor of Harsanyi (1955) and Debreu (1960), to infinite populations.

9. Conclusion. This paper has presented the following new results.

(1) Additive utility theory (Debreu 1960, Krantz et al. 1971) has been extended to infinite dimension.

(2) By means of (1), a state-dependent extension of Savage's (1954) expected utility has been obtained. We have not invoked additional restrictions such as assumptions outside the decision theoretical domain or a measure on the state space.

(3) By means of (2), it has been argued that the sure-thing principle, rather than prior probability, is at the heart of Bayesian statistics.

(0) The extension of Debreu's (1960) additive utility requires an additional innovation that should precede Step (1): The appropriate functional has to be introduced. It generalizes integration naturally, with pointwise monotonicity excluding anomalies. With that functional available, state-dependent utility can be studied without invoking additional structure outside the realm of decision theory. 
Appendix A. Extensions and further comments. In this appendix, mathematical extensions of our theorems are provided.

OBSERVATION 15. The following additions and modifications are possible in Theorem 11.

(a) The sure-thing principle in (4) of Statement (ii) need only be imposed on $\mathscr{F}^{s}$.

(b) The $V_{A} S$ in (i) are supnorm-continuous.

(c) In (i), additivity of $V$ need only be imposed on $\mathscr{F}^{s}$ and then implies additivity on all of $\mathscr{F}$.

(d) If (i) or (ii) holds, then for a null event $A, g_{A} f \sim h_{A} f$ for all acts $f, g, h$ (including the nonsimple ones).

(e) If (ii) holds, then strict monotonicity holds on all of $\mathscr{F}$, i.e., $x_{A} f>y_{A} f$ whenever $x$ $>y$ and $A$ is nonnull, also if the acts are nonsimple.

(f) The nonatomicity condition can be weakened by only requiring the existence of three disjoint nonnull events.

(g) The domain of preference need not be the entire set $\mathscr{F}_{F}$ but can be any set between $\mathscr{F}_{F}$ and $\mathscr{F}^{s}$ such that, for each element $f$ and event $A$, also $f_{A} 0$ is contained.

The results in (a), (c), (d), and (e) illustrate conditions that, when only imposed on the simple acts, extend to the nonsimple acts. In particular, the result in (d) shows that our definition of null events, which only concerns simple acts, is equivalent to the common definitions of null events that concern all acts.

The generalization in (f) covers not only the finite case considered in Theorem 2 but also infinite state spaces with atoms. We chose the nonatomic case in our main result because it is closest to Savage's model, the model that has received most attention in the literature. In Savage's (1954) expected utility model, a somewhat stronger condition was implied, i.e., that the finitely additive probability measure should be atomless: for each event $A$ and each 0 $<x<P(A)$ there should exist a subevent $B$ of $A$ with $P(B)=x$. If $P$ is countably additive, that condition is equivalent to our nonatomicity condition.

The domain restriction described in ( $\mathrm{g}$ ) generalizes the requirement of Grodal and Mertens (1976) and Vind (1990) that the domain be an "independent mixture" and contain all constant acts, which in particular generates all simple acts. Their connectedness condition implies (by Vind's Theorem III.1.1) our simple-continuity and boundedness conditions (by Vind's Remark following Corollary V.3.4). The extension of our results to nonreal outcomes is described in Appendix B.

Appendix B. Connected topological outcome spaces. This appendix demonstrates how the outcome set can be generalized to any connected topological space. This generalization is similar to Appendix 2 in Wakker (1994) and Appendix B in Chew and Wakker (1996). Also Grodal and Mertens (1976) consider outcomes more general than monetary. In their approach, the outcome set should be a separable metric space and connectedness is imposed directly on the preference topology.

$\sum_{j=1}^{n} x_{j} 1_{A_{j}}$ still denotes the simple act assigning outcome $x_{j}$ to $A_{j}$ for each $j$. The natural ordering $\geq$ on outcomes is replaced by the preference relation $\geqslant$ restricted to the constant acts, for instance in the definitions of strict and pointwise monotonicity and in the definition of strictly increasing functions. Consequently, equalities $x=y$ are sometimes replaced by equivalences $x \sim y$. $f$ from $S$ to the outcome set is bounded if there exist outcomes $x, y$ such that $[\forall s \in S: x \leqslant f(s) \leqslant y]$. Our definition of bounded can be somewhat more restrictive than the common term bounded, for instance if the outcome set is ]0, 1[. Measurability now requires that the inverse of each "preference interval" is an event. A preference interval is a subset of the outcome set that contains, for each $x \leqslant z$, also each element $y$ such that $x$ $\leqslant y \leqslant z$. Our measurability condition is ensured, in the presence of continuity of $\geqslant$, by measurability with respect to any algebra containing the topology on the outcome set. If $S$ is 
endowed with a $\sigma$-algebra, then it suffices that all inverses under $f$ of $\{x: x \geqslant y\}$ and $\{x$ $: x \leqslant y\}$ are events. $f$ from $S$ to the outcome set is an act if it is bounded and measurable.

Certainty equivalents need no longer be uniquely determined. Throughout the following analysis, it will never matter which of several certainty equivalents is chosen. Supnormcontinuity cannot be defined in this general setting. The implications of supnorm-continuity, used in the analysis, must therefore be imposed explicitly. First, simple-continuity is required, which is similarly defined as in the main text (endowing each finite product of outcome spaces with the product topology). Second, pointwise monotonicity is required. Third, the existence of a certainty equivalent is imposed for each act. Fourth and finally, a "simple-act denseness" condition is imposed to guarantee that the $V$-value of an act is indeed the infimum of the $V$-values of pointwise dominating simple acts, as well as the supremum of pointwise dominated simple acts: Simple-act denseness holds if, for all acts $f>h$, there exists a simple act $a$ such that $f>a>h$, where $a$ dominates $h$ pointwise, and a simple act $b$ such that $f>b>h$ where $b$ is pointwise dominated by $f$. The same condition is defined correspondingly for the representing functional $V$.

THEOREM 16. Let the Structural Assumption 1 hold, with the following modification: The outcome set need not be $\mathbb{R}$ but can be any connected topological space. Assume that $S$ contains no atoms. Then the following two statements are equivalent.

(i) $\geqslant$ is represented by a functional $V: \mathscr{F} \rightarrow \mathbb{R}$ that is additive, i.e., there exist functionals $V_{A}$ defined on the restrictions of acts to $A$ such that

$$
V(f)=\sum_{j=1}^{n} V_{A_{j}}\left(f_{A_{j}}\right)
$$

for each finite partition $\left\{A_{1}, \ldots, A_{n}\right\}$ of $S$. For each $A, V_{A}(x)$ (for outcome/constant act $x$ ) is continuous in $x$ and either constant or strictly increasing in $x$, and $V$ satisfies pointwise monotonicity and simple-act denseness.

(ii) $\geqslant$ is a strictly monotonic weak order that satisfies pointwise monotonicity, simplecontinuity, the existence-of-certainty-equivalent condition, simple-act denseness, and the sure-thing principle.

The uniqueness for (i) is the same as in Theorem 11. The modifications of Observation 15, except (b), also hold true for this theorem (in ( $\mathrm{g})$, let any nonmaximal outcome $\beta$ play the role of zero).

The similar adaptation of Theorem 12 is more easily formulated because pointwise continuity can still be defined. The result refers to the order topology on the outcome set, and additionally needs topological separability.

THEOREM 17. Theorem 12 also holds true for general outcome sets with a connected separable order topology.

Ordinal state independence is entailed by strict monotonicity and pointwise monotonicity. We argued in the main text that in the case of monetary outcomes there is little interest in violations of ordinal state independence. This is different for general outcomes. If outcomes are commodity bundles, for instance, then ordinal state dependence may be economically meaningful (Karni 1993a, §4.2). The extension of our representation to that case, in a way that avoids the generality of Example 4, is a topic for future research.

\section{Appendix C. Proofs of results in \$§1-6.}

Proof of Proposition 3. A partition of $S$ is essential if it contains at least three nonnull events. Note that any refinement of an essential partition is again essential because a partition 
of a nonnull event must itself contain at least one nonnull event. Obviously, an essential partition exists because of nonatomicity. To any essential partition $\pi=\left\{A_{1}, \ldots, A_{n}\right\}$, we can apply Theorem 2 to obtain a continuous additive representation $\sum_{j=1}^{n} x_{j} 1_{A_{j}} \mapsto$ $\sum_{j=1}^{n} V_{A_{j}}^{\pi}\left(x_{j}\right)$. For all essential partitions $\pi$, we can set $V_{A_{j}}^{\pi}(0)=0$ for all $A_{j}$ and $\sum_{j=1}^{n} V_{A_{j}}^{\pi}(1)$ $=1$. (Dependence of $n$ on $\pi$ is not expressed in the notation.) By considering, for any two essential partitions, the common refinement, it now follows from the uniqueness result of Theorem 2 that the additive representations of different essential partitions coincide on common domain. This also implies that, for each event $A$, the function $V_{A}^{\pi}$ is the same for every essential partition $\pi: V_{A}^{\pi}(x)$ is the value of $x 1_{A}$ in any (other) essential partition that contains $A$ or a number of events $A_{j}$ whose union is $A$. Hence, we can drop the superscript $\pi$ in $V_{A}^{\pi}$ and write $V_{A} . \sum_{j=1}^{n} x_{j} 1_{A j} \mapsto \sum_{j=1}^{n} V_{A j}\left(x_{j}\right)$ represents $\geqslant$, first only for simple acts that are measurable with respect to a same essential partition of $S$, next for all simple acts because for each pair of simple acts there is an essential partition with respect to which both acts are measurable.

By Theorem 2, all functions $V_{A}$ are continuous. Obviously, if $A$ is null, then $V_{A}$ is constant. If $A$ is nonnull, then strict monotonicity of $\geqslant$ implies that $V_{A}$ is strictly increasing.

For the uniqueness result, it is obvious that any $W$ as described can be substituted for $V$. Next, it is assumed that $W$ is additively decomposable as described and represents $\geqslant$. By Wakker (1988), $W$ and each $W_{A}$ are continuous. We can replace each $V_{A}$ by $V_{A}-V_{A}(0)$ and each $W_{A}$ by $W_{A}-W_{A}(0)$, that is, it can be assumed that $V_{A}(0)=0=W_{A}(0)$ for each event $A$. We can divide each $V_{A}$ by $V(1)$ and each $W_{A}$ by $W(1)$, i.e., it can be assumed that $V(1)$ $=1=W(1)$. It can be inferred from the proof that $W_{A}=V_{A}$ for all events $A$. In particular, $W=V$. For completeness, explicit expressions are given for the parameters $\sigma$ and $\tau$ that result from the preceding operations and that imply additivity of $\tau$ :

$$
\sigma=\frac{W(1)-W(0)}{V(1)-V(0)}, \quad \tau_{A}=W_{A}(0)-\sigma V_{A}(0) . \quad Q E D
$$

Proof of Lemma 5. Additivity of $V$ immediately implies the form described in the lemma. We now assume the form in the lemma and derive additivity. In order to do so, the definition of $V_{A}^{*}$ is extended from the acts that are constant on $A$ to arbitrary, nonconstant, restrictions of simple acts to $A$. For any simple act $f$ and event $A$, there exists a partition $\left\{B_{1}, \ldots, B_{m}\right\}$ of $A$ such that $f$ is constant and is equal to, say, $f_{j}$, on each $B_{j}$. Define $V_{A}\left(f_{A}\right)$ $=\sum_{j=1}^{m} V_{B_{j}}^{*}\left(f_{j}\right) . V_{A}$ extends $V_{A}^{*}$ to nonconstant simple acts on $A$.

Proof OF Lemma 8. First an immediate corollary of the representation in Proposition 3 is noted.

\section{Corollary 18. On $\mathscr{F}^{s}, V$ and $\geqslant$ satisfy pointwise monotonicity.}

Take any $f \in \mathscr{F}$. Because all acts are bounded in this paper, there exist outcomes $x, y$ such that $x \geq f(s) \geq y$ for all states $s$. Note that there is no monotonicity assumption to guarantee that $x \geqslant f \geqslant y$. Instead, we derive these preferences from supnorm-continuity in conjunction with strict monotonicity. It is well known that $\mathscr{F}^{s}$ is supnorm dense in $\mathscr{F}_{\mathcal{P}}$, and that we can find sequences of simple acts $g^{j}$ and $h^{j}$ such that both sequences converge in supnorm to $f$ and $g^{j}(s) \geq g^{j+1}(s) \geq f(s) \geq h^{j+1}(s) \geq h^{j}(s)$ for all states $s$. We can "truncate" every $g^{j}$ at the outcomes above $x$ and every $h^{j}$ at the outcomes below $y$, i.e., it can be assumed that $x \geq g^{j}(s)$ $\geq h^{j}(s) \geq y$ for all states $s$. According to Corollary 18, $x \geqslant g^{j} \geqslant h^{j} \geqslant y$ for all $j$. As a consequence of supnorm-continuity, $x \geqslant f \geqslant y$ follows. Consequently, the sets $\{z \in \mathbb{R}$ : $z \geqslant f\}$ and $\{z \in \mathbb{R}: z \leqslant f\}$ are nonempty. They are closed by supnorm-continuity. Because of connectedness of $\mathbb{R}$, their intersection must contain at least one element. (Because of strict monotonicity, the intersection can contain at most one element.) The element in that intersection is the certainty equivalent of $f$. 
LeMma 19. $\quad V$, defined after Lemma 8, satisfies supnorm-continuity.

Proof. Consider $\{f \in \mathscr{F}: V(f) \geq \alpha\}$ for some real $\alpha$. It will be shown that the set is closed. That is obvious if $\alpha$ is larger or smaller than any value in $V(\mathscr{F})$. In the other case, there exists a constant act $\mu$ such that $V(\mu)=\alpha$ because, by Lemma $8, V(\mathscr{F})=V(\mathbb{R})$ and the latter is connected by continuity (Proposition 3). Now $\{f \in \mathscr{F}: V(f) \geq \alpha\}=\{f$ $\in \mathscr{F}: f \geqslant \mu\}$, which is closed because of continuity of $\geqslant$. Similarly, $\{f \in \mathscr{F}: V(f) \leq \alpha\}$ is closed. Continuity of $V$ follows.

Proof of Lemma 9. Pointwise monotonicity on $\mathscr{F}^{s}$ follows from Proposition 3. Assume $f(s) \geq g(s)$ for all $s$, for two general (possibly nonsimple) acts $f, g$. It is proved that $f \geqslant g$. Take a sequence $a^{j}$ of simple acts converging pointwise from above to $f$ such that $f(s)$ $+1 / j \geq a^{j}(s) \geq a^{j+1}(s) \geq f(s)$ for all $s$ and $j$. Similarly, take a sequence $b^{j}$ of simple acts converging pointwise from below to $g$, i.e., $g(s) \geq b^{j+1}(s) \geq b^{j}(s) \geq g(s)-1 / j$ for all $s$ and $j$. We have $a^{j}(s) \geq b^{j}(s)$ for all states $s$, therefore, because of pointwise monotonicity on $\mathscr{F}^{s}$ as previously established, $V\left(a^{j}\right) \geq V\left(b^{j}\right)$ for all $j$. Because of supnorm-continuity, $V(f)=\lim V\left(a^{j}\right)$ and $V(g)=\lim V\left(b^{j}\right)$, therefore $V(f) \geq V(g)$.

Proof of Lemma 10. Assume that $V_{A}$ is defined on the restrictions of all simple acts to $A$ (as $V_{A^{*}}$ in Lemma 5). Thus, $V$ is additive on $\mathscr{F}^{s}$. Note that $V(f)-V(0)=\sum_{j=1}^{n}\left(V_{A_{j}}\left(f_{A_{j}}\right)\right.$ $\left.-V_{A_{j}}\left(0_{A_{j}}\right)\right)$ for all simple acts $f$ and partitions $\left\{A_{1}, \ldots, A_{n}\right\}$. Therefore, we can define $W_{A}$ $=V_{A}-V_{A}\left(0_{A}\right)$ for all events $A$ and restrictions of simple acts to $A$, and $W=V-V(0)$ for all acts including the nonsimple ones. Then $W$ inherits all the relevant properties of $V$, in particular additivity on $\mathscr{F}^{s}$, and in addition $W$ and all $W_{A}$ s assign 0 to the act that is constant 0 . The definition of $W_{A}$ is extended to restrictions of nonsimple acts by defining $W_{A}\left(f_{A}\right)$ $=W\left(f_{A} 0\right)$ for all acts $f$. Note that this definition agrees with the original one on $\mathscr{F}^{s}$. It is shown that with these definitions, $W$ also satisfies additivity for the nonsimple acts. Let $f$ be an arbitrary nonsimple act, $\left\{A_{1}, \ldots, A_{n}\right\}$ an arbitrary finite partition of $S$, and $f^{j}$ a sequence of simple acts converging to $f$ in supremum norm. Taking limits for $j \rightarrow \infty$, and explaining the equalities later,

$$
\begin{aligned}
W(f) & =\lim W\left(f^{j}\right)=\lim \sum_{i=1}^{n} W_{A_{i}}\left(f_{A_{i}}^{j}\right)=\sum_{i=1}^{n} \lim W_{A_{i}}\left(f_{A_{i}}^{j}\right)=\sum_{i=1}^{n} \lim W\left(f_{A_{i}}^{j} 0\right) \\
& =\sum_{i=1}^{n} W\left(f_{A_{i}} 0\right)=\sum_{i=1}^{n} W_{A_{i}}\left(f_{A_{i}}\right) \quad \text { results. }
\end{aligned}
$$

Here the first equality follows from supnorm-continuity of $W$, the second from additivity of $W$ on $\mathscr{F}^{s}$, the third mainly from boundedness of acts, the fourth from the definition of $W_{A i}$, the fifth because $f_{A_{i}}^{j} 0$ converges to $f_{A_{i}} 0$ in supnorm (implied by convergence of $f^{j}$ to $f$ ) and $W$ is supnorm-continuous, and the final equality by definition of $W_{A_{i}}$. Thus, additivity of $W$ has been established. Finally, additivity of $V$ is established. We already have $V=W+V(0)$ for all acts and $V_{A}=W_{A}+V_{A}\left(0_{A}\right)$ for all simple acts. Define $V_{A}$ similarly for all nonsimple acts. Additivity of $V$ now follows from additivity of $W$.

PROOF OF THEOREM 11. The implication (i) $\Rightarrow$ (ii) is elementary (the sure-thing principle follows from Observation 6). Next suppose (ii) holds; (i) is derived. Supnorm-continuity of $\geqslant$ implies simple-continuity because for each $n$ the supnorm topology coincides with the Euclidean topology on $\mathbb{R}^{n}$. Therefore, the representation in Proposition 3 is obtained, with each $V_{A}(x)$ continuous in $x$. By Lemma $5, V$ is additive on $\mathscr{F}^{s} . V$ is extended to all of $\mathscr{F}_{F}$ as described after Lemma 8 . It represents the preference relation, is supnorm-continuous by 
Lemma 19, and is additive by Lemma 10. $V_{A}$ is constant if $A$ is null and, because of strict monotonicity, $V_{A}(x)$ is strictly increasing in outcomes/constant acts $x$ if $A$ is nonnull. This proves the implication (ii) $\Rightarrow$ (i). By Lemma $9, V$ in (i) satisfies pointwise monotonicity. If (ii) holds, then (i) holds with $V$ pointwise monotonic, which implies that $\geqslant$ is pointwise monotonic. Continuity of $V_{A}(x)$ is ensured by Proposition 3 and its uniqueness result.

Finally, the uniqueness result is discussed, which is similar to the result of Proposition 3, the main exception being that the functions $V_{A}$ and $W_{A}$ apply not only to outcomes/constant acts on $A$ but also to nonconstant restrictions of acts to $A$. The proof is similar to the proof of the uniqueness result in Proposition 3. Note that, once the normalized functionals $V$ and $W$ agree on all simple acts, they also agree on the nonsimple acts through their certainty equivalents.

\section{Appendix D. Proofs of results in \$7.}

ProOF OF THEOREM 12. For a countably additive measure, the integral of a positive function over a nonnull set is positive. Further, if the integral of one function over every nonnull set dominates the integral of a second function, then the first function dominates the second almost everywhere. These two givens are applied on a number of occasions without further mention.

We first assume Statement (i) and prove Statement (ii). Weak ordering is immediate and the sure-thing principle follows from additivity of the representation, as in Observation 6. For strict monotonicity, assume that $x>y$ and that $A$ is not null. The latter implies, by the integral representation, that $\mu(A)>0$ must hold. For each $s \in A, U_{s}(x)>U_{s}(y)$. Because of countable additivity, $\int_{A} U_{s}(x) d \mu-\int_{A} U_{s}(y) d \mu>0$. This implies that $x_{A} f>y_{A} f$ for all acts $f$, in particular those which are simple. Strict monotonicity holds. Finally, it is well-known that continuity of a representing function implies the same continuity of $\geqslant$. Statement (ii) has been proved.

In the rest of this proof, Statement (ii) is assumed and (i) and the uniqueness results are derived.

\section{LEMMA $20 . \geqslant$ satisfies supnorm continuity.}

Proof. If a sequence of acts converges to a limiting act in supnorm, then it also converges pointwise. The boundedness of all acts in our analysis and therefore of the limiting act in particular, as well as the supnorm convergence of the sequence, imply that the sequence is uniformly bounded. By pointwise continuity, the sequence of acts converges preferencewise, which is what supnorm continuity requires. $Q E D$

By the lemma, all conditions of Statement (ii) of Theorem 11 are satisfied. Thus Statement (i) of that theorem is also satisfied, yielding the additive representation $V$.

\section{LEMMA 21. V satisfies pointwise continuity.}

Proof. Let $f^{j}$ be uniformly bounded and converge to $f$ pointwise. First assume, for contradiction, that for a subsequence of the $f^{j}$ s, the $V$ value would always exceed $V(f)$ $+\epsilon$ for a fixed positive $\epsilon$. Because of continuity, we can find a constant act $x$ with $V(f)<V(x)$ $<V(f)+\epsilon$. Pointwise continuity of $\geqslant$, and $f^{j} \geqslant x$ for all $f^{j}$ from the subsequence, imply that $f \geqslant x$, contradicting $V(f)<V(x)$. Hence, no such subsequence can exist. Similarly, no subsequence can exist for which the $V$ value would always be below $V(f)-\epsilon$ for a fixed positive $\epsilon$. Hence, $V\left(f^{j}\right)$ must converge to $V(f)$ and $V$ is pointwise continuous. $Q E D$

$V$ is normalized by subtracting $V_{A}(0)$ from all functions $V_{A}$, then dividing all those functions by the positive scale factor $V(1)$. In other words, it is assumed henceforth that $V_{A}(0)=0$ for all events $A$ and $V(1)=1$. Define $\mu(A)=V\left(1_{A}\right)$ for all $A$. It was just as possible to take any other outcomes $\alpha>\beta$, and define an alternative $\mu^{\prime}$ by $\mu^{\prime}(A)$ $=\left(V\left(\alpha 1_{A}-\beta 1_{A c}\right)\right) /(V(\alpha)-V(\beta))$. The choice of $\alpha=1$ and $\beta=0$ was arbitrary. 
LEMMA 22. For any act $f, \mu_{f}(A)=V\left(f 1_{A}\right)$ (with $\left.f 1_{A}=f_{A} 0\right)$ is a countably additive signed measure. In particular, $\mu(A)=V\left(1_{A}\right)$ is a countably additive measure.

PRoOF. $\quad \mu_{f}$ is finitely additive because $V$ is additive (hence, $\mu_{f}(\varnothing)=0$ ). For countable additivity, let $\left(A_{j}\right)_{j=1}^{\infty}$ be a sequence of disjoint events and let $A$ be their union. Then $f 1_{\cup_{j=1 A j}^{n}}$ converges to $f 1_{A}$ pointwise. The sequence is also uniformly bounded because $f$ is bounded. The $V$ values of the sequence must converge, because of pointwise continuity (Lemma 21). This means that $\sum_{j=1}^{n} \mu_{f}\left(A_{j}\right)$ converges to $\mu_{f}(A)$, and countable additivity holds. $\mu$ is a measure because pointwise monotonicity implies that it is nonnegative. (Note that also $\mu(S)$ = 1.) $Q E D$

The following lemma immediately follows from strict monotonicity.

LEMMA 23. $\mu(A)=0$ if and only if $A$ is null. $Q E D$

Because of this lemma, "null" event can equivalently refer to the preference definition as well as to the measure $\mu$. In the remainder of this proof, a.e. (almost everywhere) means that a condition holds up to a null event.

We next turn to the most complicated part of the proof, the derivation of the statedependent utility $U_{s}$. On the basis of Lemma 23 and Observation 15(d), each $\mu_{f}$ is absolutely continuous with respect to $\mu$. According to the theorem of Radon-Nikodym, we can define a density $\delta_{f}$, unique up to a null event, such that $V_{A}(f)=\int_{A} \delta_{f}(s) d \mu$ for all $A$.

First a brief preview of the proof. We will relate the definition of state-dependent utility $U_{s}(\alpha)$ to the constant act $\alpha$ by defining $U_{s}(\alpha)=\delta_{\alpha}(s)$, for all $s, \alpha$. It then remains to be verified that also for the nonconstant acts $f, \delta_{f}(s)=U_{s}(f(s))$. That is, $\delta_{f}$ should satisfy a kind of "separability" ( $\simeq$ sure-thing principle) in the sense that $\delta_{f}(s)$ depends on $f$ only through $f(s)$, and is independent of $f$ on $\{s\}^{c}$. That "state-wise" separability is ensured for simple acts by the "event-wise" separability induced by the additive representation, as will be demonstrated in Lemma 25 . An additional complication can arise for nonsimple acts. This complication is illustrated in Example 24. Following the example, state-dependent utilities are defined such that the complication is avoided. Only after that will the integral representation be established, first for simple acts, then for general.

At this point an explanation of the previously mentioned complication is in order. Each $\delta_{f}$ is only defined a.e. Therefore, $\delta_{f}$ can be considered an equivalence class of functions differing only on a null event. An appropriate representative from each such equivalence class will have to be chosen with some care when nonsimple acts are involved.

EXAmple 24. Assume that $S=[0,1]$ and that $\geqslant$ maximizes expected value. For each real $\alpha$ a representative $\delta_{\alpha}(s)=\alpha$ has been chosen, with the only exception being that for each $0 \leq \alpha \leq 1$ we have $\delta_{\alpha}(s)=0$ for $s$ in the null event $\{\alpha\}$. Then $U_{s}(\alpha)=0$ for $\alpha$ $=s$ and the act $f(s)=s$ would be assigned the state-dependent expected utility value 0 , even though $f$ is strictly preferred to the constant 0 act. Thus, state-dependent expected utility would not represent preference. In addition, each $U_{s}$ would not be strictly increasing. $Q E D$

For each real $\alpha \geq \beta$ and each event $A, \int_{A} \delta_{\alpha}(s) d \mu=V_{A}(\alpha) \geq V_{A}(\beta)=\int_{A} \delta_{\beta}(s) d \mu$, hence

$$
\alpha \geq \beta \Rightarrow \delta_{\alpha}(s) \geq \delta_{\beta}(s) \quad \text { a.e. }
$$

We first ensure that the implication in (7) holds everywhere. There are countably many pairs of rational numbers, hence there is one null event (the union of countably many) such that outside that event, (7) holds everywhere for all pairs of rational numbers. We can therefore choose the $\delta_{\rho}$ s for rational numbers $\rho$ such that (7) holds everywhere for the rational numbers. (For example, we can redefine $\delta_{\rho}(s)=\rho$ on the previously mentioned null event.)

For each real number $\alpha$, there is one null event (the union of countably many) such that 
(7) holds outside the null event for all rational $\beta$. Hence, we can choose each $\delta_{\alpha}$ such that (7) holds for all real $\alpha$ and rational $\beta$. Similarly, we can let (7) hold for all rational $\alpha$ and real $\beta$ as well. Given that for each real $\alpha>\beta$ we can find a rational number between them, we conclude that (7) now holds everywhere for all real numbers $\alpha, \beta$.

For each $s$ and outcome $\alpha$, we define $U_{s}(\alpha)=\delta_{\alpha}(s)$. By (7) as just extended, this function is nondecreasing in $\alpha$ for each $s$. We prove that the function is strictly increasing a.e. by showing that a violation would translate into a violation of the same condition for $V_{A}$ for a nonnull event $A . V$ being nondecreasing is often used in the following reasonings.

To prove that $U_{s}$ is strictly increasing a.e., consider, for any rational $\rho>\rho^{\prime}, A=\{s$ $\left.\in S: U_{s}(\rho)=U_{s}\left(\rho^{\prime}\right)\right\}$. Then $V_{A}(\rho)=V_{A}\left(\rho^{\prime}\right)$, which implies that $A$ must be null, $V_{A}$ being strictly increasing for each nonnull $A$. As there are only countably many pairs of rational numbers $\rho>\rho^{\prime}, U_{s}$ is strictly increasing in the rational numbers, hence in the real numbers, a.e. We may assume that $U_{s}$ is strictly increasing for all $s$.

LeMma 25. If $g$ is simple, then for each $A, V_{A}(g)=\int_{A} U_{s}(g) d \mu$.

PRoof. For $g, A$, there is a partition $\left\{A_{1}, \ldots, A_{n}\right\}$ of $A$ such that $g 1_{A}=\sum_{j=1}^{n} g_{j} 1_{A_{j}}$ for outcomes $g_{j}$. Now $V_{A}(g)=\sum_{j=1}^{n} V_{A_{j}}\left(g_{j}\right)=\sum_{j=1}^{n} \int_{A_{j}} \delta_{g j}(s) d \mu=\sum_{j=1}^{n} \int_{A_{j}} U_{s}\left(g_{j}\right) d \mu$ $=\int_{A} U_{s}(g) d \mu$, which is what should be proved. $Q E D$

Finally, consider a general act $f$. For each event $A$ and simple act $g$ that is pointwise dominated by $f$,

$$
\int_{A} \delta_{f}(s) d \mu=V_{A}(f) \geq V_{A}(g)=\int_{A} \delta_{g}(s) d \mu=\int_{A} U_{s}(g(s)) d \mu .
$$

Because each $U_{s}$ is strictly increasing and because of pointwise dominance, also $\int_{A} U_{s}(f(s)) d \mu \geq \int_{A} U_{s}(g(s)) d \mu$. Reversed inequalities hold both for $\int_{A} \delta_{f}(s) d \mu$ and for $\int_{A} U_{s}(f(s)) d \mu$ if $g$ pointwise dominates $f$. Because of supnorm continuity of $V_{A}$, the upper and lower bounds derived from pointwise dominating/dominated simple acts $g$ are tight and hence, for each event $A, \int_{A} U_{s}(f(s)) d \mu$ and $\int_{A} \delta_{f}(s) d \mu$ are bounded by the same upper and lower bounds, i.e., they are identical. This implies that $\delta_{f}(s)=U_{s}(f(s))$ a.e., that is, $\delta_{f}(s)=U_{s}(f(s))$ can be chosen for all $s$, and $V(f)=\int_{s} U_{s}(f(s)) d \mu$ holds for all acts $f$. Statement (i) has been proven.

Finally, the uniqueness results of the theorem are established. It is obvious that $\left(U_{s}\right)_{s \in S}, \mu$ can be replaced by $\left(U_{s}^{*}\right)_{s \in S}, \mu^{*}$ as described in the theorem. Let us now explain that no other substitutions are possible. Assume that $\left(U_{s}\right)_{s \in S}$ and $\mu$ can be replaced by $\left(U_{s}^{*}\right)_{s \in S}$ and $\mu^{*}$. Absolute continuity follows from the following lemma, which extends Lemma 23 to general $\mu^{*}$, not just the special $\mu$ as constructed in this proof.

Lemma 26. $\mu^{*}(A)=0$ if and only if $A$ is null.

Proof. If $\mu^{*}(A)=0$ then $A$ is null. Assume therefore that $\mu^{*}(A)>0$. It is shown that $A$ is nonnull. Take outcomes $x>y$. Because $U_{s}^{*}$ must be strictly increasing for each $s$ and because of countable additivity, $\int_{A} U_{s}^{*}(x) d \mu^{*}>\int_{A} U_{s}^{*}(y) d \mu^{*}$ and $A$ is nonnull. $Q E D$

Lemmas 23 and 26 imply that $\mu$ and $\mu^{*}$ have the same null events and are, therefore, absolutely continuous with respect to each other. Next we turn to the uniqueness result regarding $U$. Let $\delta$ be the Radon-Nikodym density function of $\mu$ with respect to $\mu^{*}$. We can replace $\mu^{*}$ by $\mu$ and divide $U_{s}^{*}$ by $\delta(s)$, that is, it can be assumed that $\mu^{*}=\mu$; note that $\delta$ is zero only on a null set which can be ignored. The constant $U_{s}(0)$ can be subtracted from $U_{s}(\cdot)$ and the constant $U_{s}^{*}(0)$ from $U_{s}^{*}(\cdot)$ for each $s$, i.e., it can be assumed that $U_{s}(0)=0$ $=U_{s}^{*}(0)$ for all $s$. We can divide each $U_{t}$ by the positive scale factor $\int_{s} U_{s}(1) d \mu$ and each 
$U_{t}^{*}$ by the positive scale factor $\int_{S} U_{s}^{*}(1) d \mu$, i.e., it can be assumed that these positive scale factors are 1.

It suffices to show that $U_{s}$ and $U_{s}^{*}$ agree a.e. after the preceding modifications. Consider, for any $A$ such that both $A$ and $A^{c}$ are nonnull, the two-dimensional set of acts of the form $x 1_{A}+y 1_{A^{c}}$. Here, $\int_{A} U_{s}(x) d \mu+\int_{A^{c}} U_{s}(y) d \mu=V_{A}(x)+V_{A}(y)$ provides an additive and continuous representation of preference. Such a representation also results with $U^{*}$ instead of $U$. Because the two representations have been renormalized to agree at the constant 0 and 1 acts, they must agree by the common uniqueness results of additive conjoint measurement (Karni and Safra 1998). Hence, the integrals of $U_{s}$ and $U_{s}^{*}$ over all such events $A$ agree. A similar conclusion holds for nonnull events $A$ for which $A^{c}$ is null (e.g., partition $A$ into two nonnull events, etc.). It follows that $U_{s}$ and $U_{s}^{*}$ must coincide a.e., which completes the proof of the theorem.

The following lemma demonstrates that pointwise continuity is not overly restrictive because continuity of each state-dependent utility implies pointwise continuity. Whether the reversed implication holds, i.e., whether continuity of the functions $U_{s}$ in Statement (i) of Theorem 12 holds (after appropriate modification on null events), is an open question to us.

LEMMA 27. Replacing in (ii) the pointwise continuity of the integral by continuity of each $U_{s}$ implies pointwise continuity of preference (which is equivalent to that condition for the integral) and, thus, all of (i) and (ii).

Proof. Assume that $x \geqslant f_{j}(s) \geqslant y$ for all $j$ and $s$ and that $f_{j}(s)$ converges to $f(s)$ for all $s$. Then also $U_{s}\left(f_{j}(s)\right)$ converges to $U_{s}(f(s))$ for all $s . U_{s}(x)$ and $U_{s}(y)$ are integrable upper and lower bounds, hence $\int_{s} U_{s}\left(f_{j}(s)\right) d \mu$ converges to $\int_{s} U_{s}(f(s)) d \mu$ by the dominated convergence theorem of Lebesgue (Dunford and Schwartz 1958, Corollary I.III.6.16). That is, the representing integral satisfies a pointwise continuity condition that implies pointwise continuity of $\geqslant$.

\section{Appendix E. Proof of Observation 15.}

(a) The proof of (ii) $\Rightarrow$ (i) in Theorem 11 never used more of the sure-thing principle.

(b) This follows from the equality $V_{A}\left(f_{A}\right)=V\left(f_{A} 0\right)-k$ for the constant $k=V_{A^{c}}\left(0_{A^{c}}\right)$, and from supnorm continuity of $V$.

(c) With additivity of $V$ only on $\mathscr{F}^{s}$, Statement (i) still implies Statement (ii), in particular because Statement (ii) requires the sure-thing principle only on $\mathscr{F}^{s}$ (see (a)). Statement (ii), in turn, implies all of Statement (i), in particular additivity of $V$ (mainly established in Lemma 10).

(d) Because of the sure-thing principle, the preference between $g_{A} f$ and $h_{A} f$ is the same as the preference between $g_{A} 0$ and $h_{A} 0$. Because of boundedness of acts, we can take outcomes $x, y$ such that $x \geq g(s) \geq y$ and $x \geq h(s) \geq y$ for all $s \in A$. Then $x_{A} 0 \geqslant g_{A} 0$ $\geqslant y_{A} 0$ and $x_{A} 0 \geqslant h_{A} 0 \geqslant y_{A} 0$, because of pointwise monotonicity. But also $x_{A} 0 \sim y_{A} 0$, because both acts are simple and therefore this indifference follows from event $A$ being null. It also follows that $g_{A} 0$ and $h_{A} 0$ must be indifferent to these two simple acts and hence to each other. This is likewise true of $g_{A} f$ and $h_{A} f$, because of the sure-thing principle.

(e) This follows from $x_{A} 0>y_{A} 0$ and the sure-thing principle.

(f) The proofs require no modifications for this result. (In particular, the proof of Proposition 3 has been written so that it also applies to this case.)

(g) When the domain under consideration is a subset of $\mathscr{F}^{s}$, then the definition of the various conditions such as the sure-thing principle are obviously restricted to the domain under consideration. Note that, by (a) of Observation 15 , the sure-thing principle is only needed on $\mathscr{F}^{s}$. The proofs require no other modifications for this case. 
Appendix F. Proof of Theorems 16 and 17 (connected topological outcome spaces). In this appendix, the proofs of the preceding results for connected topological outcome spaces are presented.

ProOF OF THEOREM 16. The implication (i) $\Rightarrow$ (ii) is straightforward, the existence of certainty equivalents following from continuity and pointwise monotonicity of $V$, and connectedness of the outcome set, as in the proof of Lemma $8,(x \geqslant f \geqslant y$ there follows immediately from pointwise monotonicity of $V$ ). Next (ii) is assumed and (i) is derived. This is demonstrated in a similar manner as the implication (ii) $\Rightarrow$ (i) in Theorem 11 and goes through a number of steps.

(STEP 1) Theorem 2 needs no modification. Note, however, that Debreu (1960) also assumed topological separability of the outcome space. It was pointed out by Krantz et al. (1971) that this assumption can be dropped (their proof was supplemented by Wakker (1988) and by Wakker (1989, Theorem III.6.6 and Remark A3.1).

(STEP 2) In the case of Proposition 3 and the subsequent results, one difference is that no natural 0 and 1 outcomes are given. The remedy is easy: take any outcomes $\alpha>\beta$, then "normalize" $V$ by setting $V_{A}(\beta)=0$ for all $A$ and $V(\alpha)=1$. Subsequently, $\beta$ plays the role of the 0 -outcome, $\alpha$ the role of the 1-outcome. Other than that, the results in $\$ 5$ need no modification.

(STEP 3) The result of Lemma 8 is now assumed explicitly, and Lemma 9 immediately follows from the similar assumption for preference.

(STEP 4) The proof of Lemma 10 requires a more elaborate revision, concerning additivity of the functional $W$ constructed there. We cannot invoke supnorm continuity at this point, and instead must invoke simple-act denseness and the other assumptions. Let $f$ be a nonsimple act and $\left\{A_{1}, \ldots, A_{n}\right\}$ a partition of $S$. It is shown that $W(f)=\sum_{i=1}^{n} W_{A_{i}}\left(f_{A_{i}}\right)$. Let $\gamma$ be a certainty equivalent of $f$. First, sequences $a^{j}, b^{j}$ of simple acts are constructed such that (1) with $W$ the normalized version of $V$ that satisfies $W_{A}(\beta)=0$ for all $A$ and $W(\alpha)=1$, the result is

$$
W(f)-1 / j \leq W\left(b^{j}\right) \leq W(f)=W(\gamma) \leq W\left(a^{j}\right) \leq W(f)+1 / j
$$

for all $j$, (2) $f$ dominates each $b^{j}$ pointwise, and (3) each $a^{j}$ dominates $f$ pointwise. The construction of the $a^{j}$ s is described in detail. If $f$ is maximal, i.e., no act $g$ is strictly preferred to $f$ (this case can occur for general connected topological outcome spaces), then the certainty equivalent $\gamma$ of $f$ must be a maximal outcome and we simply take each $a^{j}$ equal to $\gamma$. Note that this dominates $f$ pointwise. If $f$ is not maximal, then there exists a strictly preferred act and the certainty equivalent thereof is also strictly preferred to $f$ and $\gamma$. Because of simple-continuity on $\mathscr{F}^{s}$ and connectedness, the $W$ image of outcomes is an interval. Hence, there must exist, for each $j$, a constant act $c^{j}$ which is between the certainty equivalent strictly preferred to $f$, and $\gamma$, such that $W(\gamma)=W(f)<W\left(c^{j}\right) \leq W(f)+1 / j$. Now simple-act denseness is invoked, which implies the existence of a simple act $a^{j}$ that dominates $f$ pointwise and satisfies $W(f) \leq W\left(a^{j}\right) \leq W\left(c^{j}\right) \leq W(f)+1 / j$. The acts $b^{j}$ are constructed similarly. We have now achieved the same kind of enclosure of $f$ between simple acts, from above and below, as under supnorm continuity for real outcomes.

Taking limits for $j \rightarrow \infty$, we get (explaining the equalities later)

$$
\begin{aligned}
W(f) & =\lim W\left(a^{j}\right)=\lim \sum_{i=1}^{n} W_{A_{i}}\left(a_{A_{i}}^{j}\right)=\sum_{i=1}^{n} \lim W_{A_{i}}\left(a_{A_{i}}^{j}\right)=\sum_{i=1}^{n} \lim W\left(a_{A_{i}}^{j} \beta\right) \\
& =\sum_{i=1}^{n} W\left(f_{A_{i}} \beta\right)=\sum_{i=1}^{n} W_{A_{i}}\left(f_{A_{i}}\right)
\end{aligned}
$$

establishing additivity of $W$. 
The first equality follows from the definition of the acts $a^{j}$, the second follows from additivity of $W$ on $\mathscr{F}^{s}$ (Lemma 5), the third is elementary because of boundedness, the fourth follows from the definition of $W_{A i}$, the fifth is explained later, and the sixth follows from the definition of $W_{A i}$. For the fifth equality it is shown that $\lim _{j \rightarrow \infty} W\left(a_{A_{i}}^{j} \beta\right)=W\left(f_{A_{i}} \beta\right)$ for each $i$. This follows from the inequalities $2 / j \geq W\left(a_{A_{i}}^{j} \beta\right)-W\left(f_{A_{i}} \beta\right) \geq 0$ for all $j$, which is derived from the following inequalities, explained thereafter:

$$
\frac{2}{j} \geq W\left(a^{j}\right)-W\left(b^{j}\right) \geq W\left(a_{A_{i}}^{j} \beta\right)-W\left(b_{A_{i}}^{j} \beta\right) \geq W\left(a_{A_{i}}^{j} \beta\right)-W\left(f_{A_{i}} \beta\right) \geq 0
$$

for all $j$. First, note that all differences in the inequality are nonnegative because the left act always dominates the right act pointwise. The first inequality follows from the construction of the simple acts $a^{j}$ and $b^{j}$, which both differ by less than $1 / j$ from $f$ in $W$ units. For the second inequality, we invoke the additive representation on $\mathscr{F}_{F}^{s}$ with respect to the partition $\left\{A_{1}, \ldots, A_{n}\right\}$. The term corresponding to event $A_{i}$ is the same for the left- and right-hand side, for all other events the terms for the left-hand side are nonnegative, for the right-hand side they are 0 . The third inequality follows because $f_{A i} \beta$ dominates $b_{A i}^{j} \beta$ pointwise. The fourth inequality follows because $\left(a_{A i}^{j} \beta\right)$ dominates $f_{A i} \beta$ pointwise.

Thus, additivity of the functional $W$ has been proven. Other than that, the proof needs no adaptation.

(STEP 5) Next we turn to the completion of the proof of the implication (ii) $\Rightarrow$ (i) in Theorem 16, by adapting the related proof in Theorem 11. Pointwise monotonicity and simple-act denseness are equivalent for preferences and for $V$. Supnorm continuity is not even defined now. This establishes the implication.

(STEP 6) The uniqueness results in Theorem 16, and the extensions provided in Observation 15 are proved in the same manner as in Theorem 11 (where again any $\alpha>\beta$ play the role of the outcomes 1 and 0 ).

PROOF OF THEOREM 17. The implication (i) $\Rightarrow$ (ii) is again straightforward, hence we assume (ii) and derive (i). Pointwise continuity implies simple-continuity, hence we can invoke Theorem 2 and Proposition 3. For the proof of the following lemma, it is crucial that the outcome set is endowed with the order topology.

\section{LemMa 28. Simple-act denseness holds.}

Proof. Let $Y$ denote a countable dense subset of the outcome space; such a subset exists because of topological separability. We may assume that it contains a maximal and minimal outcome if such exist. For each act $f$ we can construct dominating simple acts $a^{j}$ and dominated simple acts $b^{j}$, respectively, that converge to $f$ pointwise, as follows. Write $Y$ $=\left\{y_{1}, y_{2}, \ldots\right\}$. Let $z_{0} \in Y$ satisfy $z_{0} \geqslant f(s)$ for all $s$. Such a $z_{0}$ exists because $f$ is bounded and $Y$ contains a maximal outcome if such exists. Let $\left\{z_{1}, \ldots, z_{j}\right\}$ be a reordering of $\left\{y_{1}, \ldots, y_{j}\right\}$ such that $z_{1} \geqslant \cdots \geqslant z_{j}$. Then $a^{j}=\sum_{i=0}^{j} z_{i} 1_{A_{i}}$ where $A_{i}=\left\{s \in S: z_{i}\right.$ $\left.\geqslant f(s)>z_{i+1}\right\}$ for all $i \leq j-1, A_{j}=\left\{s \in S: z_{j} \geqslant f(s)\right\}$. Each $a^{j}$ dominates $a^{j+1}$ pointwise and they all dominate $f$ pointwise. They converge to $f$ pointwise because for each $s$ and $x>f(s)$, some $y_{k}$ will come between $x$ and $f(s)$ implying $x>y_{k} \geqslant a^{j}(s) \geqslant f(s)$ for all $j \geq k$. The $b^{j}$ s are defined similarly. Both sequences of simple acts are uniformly bounded (the $a^{j} \mathrm{~s}$ are bounded by $z_{0}$ and the lower bound of $f$ ), hence pointwise convergence implies preference-wise convergence due to pointwise continuity. That implies simple-act denseness. $Q E D$

The proof of Lemma 8 can now be adapted in a straightforward manner, using pointwise continuity instead of supnorm continuity, and using Lemma 28. Thus, for every act a certainty equivalent exists (possibly more). By pointwise continuity, the simple acts that converge to an act pointwise also converge in preference. Hence, $V$ (defined through certainty 
equivalents as in the main text) inherits pointwise monotonicity for all acts from pointwise monotonicity on the simple acts, i.e., Lemma 9 also holds. Now all conditions in Statement (ii) of Theorem 16 hold, therefore Statement (i) there also holds. From this point on, the proof of Theorem 12 from Lemma 21 onwards can be followed by fixing any $\alpha>\beta$ instead of 1 $>0$, taking a countable dense subset $Y$ instead of the rational numbers, and applying pointwise continuity instead of supnorm continuity.

\section{Appendix G. Proof of results in $\$ 8$.}

Proof OF THEOREM 13. If (i) holds, then for all $\lambda_{j}$ equal to $0, A_{j}$ is posterior-null. For all positive $\lambda_{j}$, the posterior preference relation conditional on $A_{j}$ is represented by $V_{A_{j}}$, i.e., it is identical to the prior preference relation conditional on $A_{j}$. Hence (i) implies (ii). Next assume (ii). Assume that the posterior preference relation, satisfying all requirements of Theorem 11, is represented by $f \mapsto \sum_{j=1}^{n} W_{A_{j}}\left(f_{A_{j}}\right)$. We may assume that, for each event $A$, $V_{A}(0)=0$ and $W_{A}(0)=0$. If $A_{j}$ is posterior-null then $W_{A_{j}}=0$ and we set $\lambda_{j}=0$. Next assume $A_{j}$ is not posterior-null. In this case, the prior and posterior preference relation, conditional on $A_{j}$, are the same. The crucial point in this proof is based on consideration of this preference relation on its own, and applying Theorem 11 to it. Thus $S^{\prime}=A_{j}$ is considered to be a state space. Note that it contains no atoms. The preference relation satisfies all conditions in Theorem 11, and $V_{A_{j}}$ and $W_{A_{j}}$ are additive representations of it. From the uniqueness result in Theorem 11 and the scaling $V_{A}(0)=0$ and $W_{A}(0)=0$ for all events $A \subset A_{j}$, it follows that there exists a positive factor $\lambda_{j}$ such that $W_{A_{j}}=\lambda_{j} V_{A_{j}}$.

Proof of TheORem 14. Take any finite partition $\left\{A_{1}, \ldots, A_{n}\right\}$ containing at least two nonnull events. Then the claim of the theorem, including expected utility representation, holds for acts that are constant on each element of the partition, by Miyamoto and Wakker (1996, Example 1.c). If expected utility holds on every finite partition, then in the presence of the other assumptions, it must hold for all acts (Wakker 1993, Corollary 2.14; Wakker's assumption of truncation-continuity is vacuously satisfied in our context where all acts are bounded).

\section{References}

$\rightarrow$ Anscombe, F. J., R. J. Aumann 1963. A definition of subjective probability. Ann. Math. Statist. 34 199-205.

Barten, A. P., V. Böhm 1982. Consumer theory. K. J. Arrow and M. D. Intriligator eds. Handbook of Mathematical Economics II, Chapter 9 381-429. North-Holland, Amsterdam.

Broome, J. 1991. Weighing Goods. Basil Blackwell, Oxford, UK.

Chateauneuf, A. 1991. On the use of capacities in modeling uncertainty aversion and risk aversion. J. Math. Econom. 20 343-369.

Chew, S. H., P. P. Wakker 1996. Generalizing Choquet expected utility by weakening Savage's sure-thing principle. J. Risk and Uncertainty 12 5-27.

Debreu, G. 1960. Topological methods in cardinal utility theory. K. J. Arrow, S. Karlin, and P. Suppes, eds. 1959. Mathematical Methods in the Social Sciences, 16-26, Stanford University Press, Stanford, CA.

Drèze, J. 1961. Les fondements logiques de l'utilité cardinale et de la probabilité subjective. La Décision 73-83 CNRS, Paris, France.

1987. Essays on Economic Decision under Uncertainty. Cambridge University Press, London, UK.

Dunford, N., J. T. Schwartz 1958. Linear Operators, Part I. Interscience Publishers, New York.

Epstein, L. G., M. le Breton 1993. Dynamically consistent beliefs must be Bayesian. J. Econom. Theory 61 1-22.

Fishburn, P. C. 1970, Utility Theory for Decision Making. Wiley, New York.

$\rightarrow-1973$. A mixture-set axiomatization of conditional subjective expected utility. Econometrica 41 1-24.

$\rightarrow-1986$. The axioms of subjective probability. Statist. Sci. $1335-358$.

$\rightarrow-1992$. Utility as additive set function. Math. Oper. Res. 17 910-920.

$\rightarrow$ Fleming, J. M. 1952. A cardinal concept of welfare. Quarterly J. Econom. 66 366-384.

$\rightarrow$ Gorman, W. M. 1968. The structure of utility functions. Rev. Econom. Stud. 35 367-390.

Green, J., B. Jullien 1988. Ordinal independence in non-linear utility theory. J. Risk and Uncertainty 1355-387. (Erratum. 1989. 2 119.)

Grodal, B. 1978. Some further results on integral representation of utility functions. Institute of Economics, University of Copenhagen, Copenhagen. 
J.-F. Mertens 1976. Integral representation of utility functions. Institute of Economics, University of Copenhagen, Copenhagen, Denmark.

Gul, F. 1992. Savage's theorem with a finite number of states. J. Econom. Theory 57 99-110. (Erratum. 1993. 61 184.)

$\rightarrow$ Harsanyi, J. C. 1955. Cardinal welfare, individualistic ethics, and interpersonal comparisons of utility. J. Political Econom. 63 309-321.

Hübner, R., R. Suck 1993. Algebraic representation of additive structure with an infinite number of components. $J$. Math. Psychology 37 629-639.

$\rightarrow$ Kadane, J. B., R. L. Winkler 1988. Separating probability elicitation from utilities. J. Amer. Statist. Assoc. 83 357-363.

$\rightarrow$ Karni, E. 1983. Risk aversion for state-dependent utility functions: Measurement and applications. Internat. Econom. Rev. 24 637-647.

1985. Decision-Making under Uncertainty: The Case of State-Dependent Preferences. Harvard University Press, Cambridge, MA.

1993a. A definition of subjective probabilities with state dependent preferences. Econometrica 61 187-198. 1993b. Subjective expected utility theory with state dependent preferences. J. Econom. Theory 60 428-438. 1996. Probabilities and beliefs. J. Risk and Uncertainty 13 249-262.

1997. Elicitation of subjective probabilities when preferences are state-dependent. Internat. Econom. Rev., forthcoming.

- Z. Safra 1998. Hexagon condition and additive representation of preferences: An algebraic approach. $J$. Math. Psychology, 42 393-399.

- D. Schmeidler 1993. On the uniqueness of subjective probabilities. Econom. Theory 3 267-277.

$\rightarrow-,-$, K. Vind 1983. On state dependent preferences and subjective probabilities. Econometrica 51 1021-1031.

Keeney, R. L., H. Raiffa 1976. Decisions with Multiple Objectives. Wiley, New York. (2nd edition, 1993. Cambridge University Press, Cambridge UK).

Klibanoff, P. 1995. Dynamic choice with uncertainty aversion. Center for Mathematical Studies in Economics and Management Science, Northwestern University, Evanston, IL.

Koopmans, T. C. 1972. Representations of preference orderings with independent components of consumption, and Representations of preference orderings over time. C. B. McGuire and R. Radner (Eds.), Decision and Organization, 57-100, North-Holland, Amsterdam.

Krantz, D. H., R. D. Luce, P. Suppes, A. Tversky 1971. Foundations of Measurement, Vol. I. (Additive and Polynomial Representations). Academic Press, New York.

Kreps, D. M. 1988. Notes on the Theory of Choice. Westview Press, Boulder, CO.

LaValle, I. H., P. C. Fishburn 1991. Lexicographic state-dependent subjective expected utility. J. Risk and Uncertainty 4 251-269.

$\rightarrow$ Luce, R. D., D. H. Krantz 1971. Conditional expected utility. Econometrica 39 253-271.

$\rightarrow$ Machina, M. J., D. Schmeidler 1992. A more robust definition of subjective probability. Econometrica 60 745-780.

Maher, P. 1993. Betting on Theories. Cambridge University Press, Cambridge, UK.

$\rightarrow$ Miyamoto, J., P. P. Wakker 1996. Multiattribute utility theory without expected utility foundations. Oper. Res. 44 313-326.

Nakamura, Y. 1990. Subjective expected utility with non-additive probabilities on finite state spaces. J. Econom. Theory 51 346-366.

Nau, R. F. 1995. Coherent decision analysis with inseparable probabilities and utilities. J. Risk and Uncertainty 10 71-91.

, K. F. McCardle 1991. Arbitrage, rationality, and equilibrium. Theory and Decision 31 199-240.

$\rightarrow$ Poirier, D. J. 1988. Frequentist and subjective perspectives on the problems of model building in economics. $J$. Econom. Perspectives 2 121-144.

Quiggin, J. 1989. Sure things-Dominance and independence rules for choice under uncertainty. Ann. Oper. Res. 19 335-357.

Rubin, H. 1949. Postulates for the existence of measurable utility and psychological probability (abstract 493). Bull. Amer. Math. Soc. 55 1050-1051.

1987. A weak system of axioms for "rational" behavior and the nonseparability of utility from prior. Statist. Decision 5 47-58.

Savage, L. J. 1954. The Foundations of Statistics. Wiley, New York. (2nd edition 1972, Dover, New York.)

$\rightarrow$ Schervish, M. J., T. Seidenfeld, J. B. Kadane 1990. State-dependent utilities. J. Amer. Statist. Assoc. 85 840-847.

$\rightarrow$ Schmeidler, D. 1989. Subjective probability and expected utility without additivity. Econometrica 57 571-587.

Segal, U. 1993. The measure representation: A correction. J. Risk and Uncertainty 6 99-107.

Streufert, P. A. 1995. A general theory of separability for preferences defined on a countably infinite product space. J. Math. Econom. 24 407-434.

$\rightarrow$ Strotz, R. H. 1956. Myopia and inconsistency in dynamic utility maximization. Rev. Econom. Stud. 23 (3) (June) 165-180. 
Vind, K. 1990. Additive utility functions and other special functions in economic theory, (with contributions by Birgit Grodal). Discussion paper 90-21. Institute of Economics, University of Copenhagen, Denmark.

Wakker, P. P. 1984. Cardinal coordinate independence for expected utility. J. Math. Psychology 28 110-117.

- 1987. Subjective probabilities for state-dependent continuous utility. Math. Social Sci. 14 289-298.

1988. The algebraic versus the topological approach to additive representations. J. Math. Psychology 32 421-435.

1989. Additive Representations of Preferences, A New Foundation of Decision Analysis. Kluwer Academic Publishers, Dordrecht, The Netherlands.

1993. Unbounded utility for Savage's "foundations of statistics," and other models. Math. Oper. Res. 18 446-485.

1994. Separating marginal utility and probabilistic risk aversion. Theory and Decision 36 1-44.

D. Deneffe 1996. Eliciting von Neumann-Morgenstern utilities when probabilities are distorted or unknown. Management Sci. 42 1131-1150.

P. P. Wakker: CentER, Tilburg University, P.O. Box 90153, Tilburg, 5000 LE, The Netherlands

H. Zank: Department of Economics, Maastricht University, P.O. Box 616, Maastricht, 6200 MD, The Netherlands 